\title{
Magnetotransport in a Model of a Disordered Strange Metal
}

\author{
Aavishkar A. Patel, ${ }^{1,2}$ John McGreevy, ${ }^{3}$ Daniel P. Arovas, ${ }^{3}$ and Subir Sachdev ${ }^{1,4,5}$ \\ ${ }^{1}$ Department of Physics, Harvard University, Cambridge, Massachusetts 02138, USA \\ ${ }^{2}$ Kavli Institute for Theoretical Physics, University of California, \\ Santa Barbara, California 93106-4030, USA \\ ${ }^{3}$ Department of Physics, University of California at San Diego, La Jolla, California 92093, USA \\ ${ }^{4}$ Perimeter Institute for Theoretical Physics, Waterloo, Ontario, Canada N2L $2 Y 5$ \\ ${ }^{5}$ Department of Physics, Stanford University, Stanford, California 94305, USA
}

(Received 30 December 2017; revised manuscript received 4 March 2018; published 22 May 2018)

\begin{abstract}
Despite much theoretical effort, there is no complete theory of the "strange" metal state of the high temperature superconductors, and its linear-in-temperature $T$ resistivity. Recent experiments showing an unexpected linear-in-field $B$ magnetoresistivity have deepened the puzzle. We propose a simple model of itinerant electrons, interacting via random couplings, with electrons localized on a lattice of "quantum dots" or "islands." This model is solvable in a particular large- $N$ limit and can reproduce observed behavior. The key feature of our model is that the electrons in each quantum dot are described by a Sachdev-Ye-Kitaev model describing electrons without quasiparticle excitations. For a particular choice of the interaction between the itinerant and localized electrons, this model realizes a controlled description of a diffusive marginal-Fermi liquid (MFL) without momentum conservation, which has a linear-in- $T$ resistivity and a $T \ln T$ specific heat as $T \rightarrow 0$. By tuning the strength of this interaction relative to the bandwidth of the itinerant electrons, we can additionally obtain a finite- $T$ crossover to a fully incoherent regime that also has a linear-in- $T$ resistivity. We describe the magnetotransport properties of this model and show that the MFL regime has conductivities that scale as a function of $B / T$; however, the magnetoresistance saturates at large $B$. We then consider a macroscopically disordered sample with domains of such MFLs with varying densities of electrons and islands. Using an effective-medium approximation, we obtain a macroscopic electrical resistance that scales linearly in the magnetic field $B$ applied perpendicular to the plane of the sample, at large $B$. The resistance also scales linearly in $T$ at small $B$, and as $T f(B / T)$ at intermediate $B$. We consider implications for recent experiments reporting linear transverse magnetoresistance in the strange metal phases of the pnictides and cuprates.
\end{abstract}

DOI: 10.1103/PhysRevX.8.021049

Subject Areas: Condensed Matter Physics, Strongly Correlated Materials

\section{INTRODUCTION}

Essentially all correlated electron high temperature superconductors display an anomalous metallic state at temperatures above the superconducting critical temperature at optimal doping [1-3]. This metallic state has a "strange," linearly increasing dependence of the resistivity $\rho$ on temperature $T$; it can also exhibit bad metal behavior with a resistivity much larger than the quantum unit $\rho \gg$ $h / e^{2}$ (in two spatial dimensions) [4]. More recently, strange metals have also been demonstrated to have a remarkable linear-in- $B$ magnetoresistance, with the crossover between

Published by the American Physical Society under the terms of the Creative Commons Attribution 4.0 International license. Further distribution of this work must maintain attribution to the author(s) and the published article's title, journal citation, and DOI. the linear-in- $T$ and linear-in- $B$ behavior occurring at $\mu_{B} B \sim k_{B} T[5,6]$.

This paper will present a model of a strange metal that exhibits the above linear-in- $T$ and linear-in- $B$ behavior. The model builds on a lattice array of "quantum dots," each of which is described by a Sachdev-Ye-Kitaev (SYK) model of fermions with random all-to-all interactions $[7,8]$. The SYK models are $(0+1)$-dimensional quantum theories that exhibit a "local criticality." They have drawn a great deal of interest for a variety of reasons:

(i) The SYK models are the simplest solvable models without quasiparticle excitations. They can also be used as fully quantum building blocks for theories of strange metals in nonzero spatial dimensions $[9,10]$.

(ii) The SYK models exhibit many-body chaos $[8,11]$ and saturate the lower bound on the Lyapunov time to reach chaos [12]. So, they are "the most chaotic" quantum many-body systems. The presence of maximal chaos is linked to the absence of quasiparticle 
excitations and the proposed [13] lower bound of order $\hbar /\left(k_{B} T\right)$ on a "dephasing time." It is important to note here that the coexistence of many-body chaos and solvability is quite remarkable: Essentially all other solvable models (e.g., integrable lattice models in one dimension) do not exhibit many-body chaos.

(iii) Related to their chaos, the SYK models exhibit [14] eigenstate thermalization (ETH) $[15,16]$, and yet many aspects are exactly solvable.

(iv) The SYK models are dual to gravitational theories in $1+1$ dimensions that have a black hole horizon. The connection between the SYK models and black holes with a near-horizon $\mathrm{AdS}_{2}$ geometry was proposed in Refs. [17,18] and made much sharper in Refs. $[8,19,20]$. This connection has been used to examine aspects of the black hole information problem [21].

More specifically, a single SYK site is a $(0+1)$ dimensional non-Fermi liquid in which the imaginary-time $(\tau)$ fermion Green's function has the low $T$ "conformal" form $[7,9,22,23]$

$G(\tau) \sim\left(\frac{T}{\sin (\pi T \tau)}\right)^{1 / 2} e^{-2 \pi \mathcal{E} T \tau}, \quad 0<\tau<1 / T$,

where $\mathcal{E}$ is a parameter controlling the particle-hole asymmetry. In frequency space, this correlator is $G(\omega) \sim 1 / \sqrt{\omega}$ for $\omega \gg T$, and this implies non-Fermi liquid behavior. A Fermi liquid has the exponent $1 / 2$ in Eq. (1.1) replaced by unity, and a constant density of states with $G(\omega)$ frequency independent. The Green's function in Eq. (1.1) implies [7] a "marginal" [24] susceptibility $\chi$, with a real part that diverges logarithmically with vanishing frequency $(\omega)$ or $T$. Specifically, in the all-to-all limit of the SYK model, vertex corrections are subdominant, and the Fourier transform of $\chi(\tau)=-G(\tau) G(-\tau)$ leads to the spectral density

$$
\operatorname{Im} \chi(\omega) \sim \tanh \left(\frac{\omega}{2 T}\right),
$$

whose Hilbert transform leads to the noted logarithmic divergence. In contrast, a Fermi liquid has $\operatorname{Im} \chi(\omega) \sim \omega$. The form in Eq. (1.2) is consistent with recent electron scattering observations [25]. A linear-in- $T$ resistivity now follows upon considering itinerant fermions scattering off such a local susceptibility, and the itinerant fermions realize a marginal Fermi liquid (MFL) with a $\omega \ln \omega$ self-energy $[7,17,24,26]$.

We now review previous approaches to building a finite-dimensional non-Fermi liquid from the $(0+1)$ dimensional SYK model. An early model for a bulk strange metal in finite spatial dimensions was provided by Parcollet and Georges [9]. They considered a doped Mott insulator described by a random $t-J$ model at hole density $\delta$, where $t$ is the root-mean-square (rms) electron hopping, and $J$ is the rms exchange interaction. At low doping with $\delta t \ll J$, they found strange metal behavior in the intermediate $T$ regime $E_{c}<T<J$, where the coherence energy $E_{c}=(\delta t)^{2} / J$. In this intermediate energy range, they found that the electron Green's function had the local form of the SYK model in Eq. (1.1). Moreover, this metal had "bad metal" resistivity with $\rho \sim\left(h / e^{2}\right)\left(T / E_{c}\right) \gg\left(h / e^{2}\right)$. We will refer to such a strange metal as an "incoherent metal" (IM). This IM is to be contrasted from a MFL, which we will describe below; the MFL does not appear in the model of Parcollet and Georges.

Another finite-dimensional model of an IM appeared in the recent work of Song et al. [10]. They considered a lattice of SYK sites, with rms on-site interaction $U$, and rms intersite hopping $t$. Each site was a quantum dot with $N$ orbitals and had random on-site interactions with typical magnitude $U$. Electrons were allowed to hop between nearest-neighbor states, with a random matrix element of magnitude $t$. Although this is a model with strong interactions, the remarkable fact is that the random nature of the interactions renders it exactly solvable. As in Ref. [9], Song et al. found an IM in the intermediate regime $E_{c}<T<U$, with a local electron Green's function, as in Eq. (1.1), and a bad metal resistivity $\rho \sim\left(h / e^{2}\right)\left(T / E_{c}\right)$. Their coherence scale was $E_{c}=t^{2} / U$. (This lattice SYK model should be contrasted from earlier studies [27,28], which only had fermion interaction terms between neighboring SYK sites: The latter models realize disordered metallic states without quasiparticle excitations as $T \rightarrow 0$, but have a $T$-independent resistivity.)

Although these models $[9,10]$ reproduce bad metal resistivity, we will show here that they are unable to describe the experimentally observed large magnetoresistance noted earlier $[5,6]$. The random nature of the hopping between the sites, and the associated absence of a Fermi surface, results in negligible magnetoresistance. Significant orbital magnetoresistance only appears in models that have fermions with nonrandom hopping and a well-defined Fermi surface. Note that the existence of a Fermi surface does not directly imply the presence of well-defined quasiparticles: It is possible to have a sharp Fermi surface in momentum space (where the inverse fermion Green's function vanishes) while the quasiparticle spectral function is broad in frequency space.

With the aim of obtaining a well-defined Fermi surface of itinerant electrons, in this paper we consider a lattice of SYK "islands" coupled to a separate band of itinerant conduction electrons, as illustrated in Fig. 1. Our model is in the spirit of effective Kondo lattice models that have been proposed as models of the physics of the disordered, single-band Hubbard model [29-31]. The other two band models of itinerant electrons coupled to SYK excitations have been considered in Refs. [32,33]. Our model exhibits MFL behavior as $T \rightarrow 0$, with a linear-in- $T$ resistivity, and 

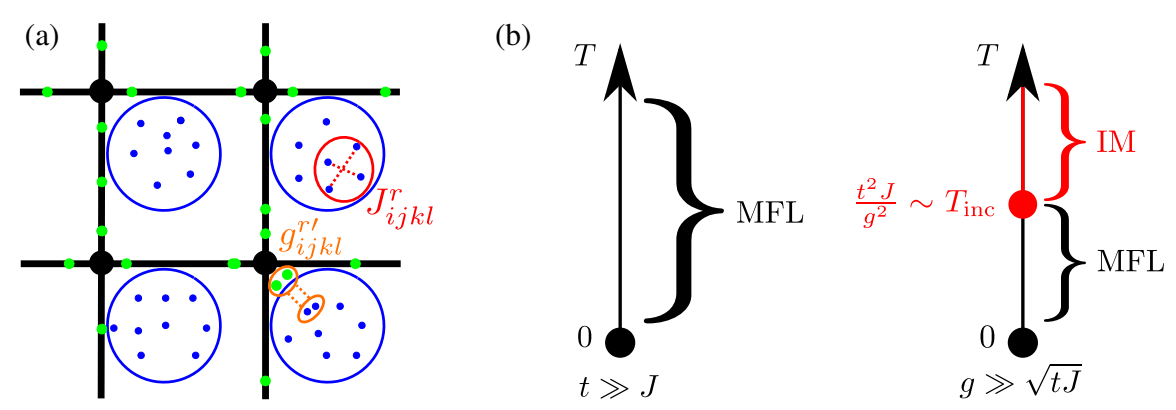

FIG. 1. (a) Our microscopic model. Itinerant conduction electrons (green) hop around on a lattice (black). At each lattice site, they interact locally and randomly with SYK quantum dots (blue) through an interaction (orange) that independently conserves the numbers of conduction and island electrons. (b) Finite-temperature regimes of the model. When the conduction electron bandwidth is large enough, it realizes a disordered marginal-Fermi liquid (MFL) for the conduction electrons for all temperatures $T \ll J$ (Sec. III A). For a finite bandwidth, there can be a finite-temperature crossover to an incoherent metal (IM), in which all notion of electron momentum is lost, if the coupling $g$ is large enough (Sec. III B). Note that we always have $J \gg T$ and $J \gtrsim g$.

a $T \ln T$ specific heat. For an appropriate range of parameters, there is a crossover at higher $T$ to an IM regime, also with a linear-in- $T$ resistivity. The itinerant electrons have a nonrandom hopping $t$, the SYK sites have a random interaction with rms strength $J$, and these two subsystems interact with a random Kondo-like exchange of rms strength $g$; see Fig. 1(a) for a schematic illustration. Figure 1(b) illustrates the regimes of MFL and IM behavior in our model. In the MFL regime, our model exhibits a well-defined Fermi surface, albeit of damped quasiparticles.

The magnetotransport properties of this model will be a significant focus of our analysis. We will show that the MFL regime with a Fermi surface indeed has a sizable magnetoresistance, with characteristics in accord with observations. We find that the longitudinal and Hall conductivities of the MFL regime can be written as scaling functions of $B / T$, as shown in Eq. (4.12). In contrast, the $B$ dependence is much less singular in the IM regime. Although a $B / T$ scaling is obtained in the MFL in this computation, the magnetoresistance does not increase linearly with $B$ and instead saturates at large $B$. To obtain a nonsaturating magnetoresistance, we consider a macroscopically disordered sample with domains of MFLs with varying electron densities; employing earlier work on classical electrical transport in inhomogeneous ohmic conductors [34-40], we obtain the observed linear-in- $B$ magnetoresistance with a crossover scale at $B \sim T$.

This paper is organized as follows: In Sec. II, we introduce our basic microscopic model of a disordered MFL, and we determine its single-electron properties and finite-temperature crossovers in Sec. III. In Sec. IV, we solve for transport and magnetotransport properties of this basic model exactly in various analytically tractable regimes. In Sec. V, we introduce the effective-medium approximation and apply it to a macroscopically disordered sample containing domains of the basic model, obtaining analytical results for the global magnetotransport properties for certain simplified considerations of macroscopic disorder. We summarize our results and place them in the context of recent experiments in Sec. VI.

\section{MICROSCOPIC MODEL}

We consider $M$ flavors of conduction electrons $c$ hopping on a lattice that are coupled locally and randomly to SYK islands on each lattice site [Fig. 1(a)]. The islands contain $N$ flavors of valence electrons $f$, which interact among themselves in such a way that they realize SYK models. The Hamiltonian for our system is given by

$$
\begin{aligned}
H= & -t \sum_{\left\langle r r^{\prime}\right\rangle ; i=1}^{M}\left(c_{r i}^{\dagger} c_{r^{\prime} i}+\text { H.c. }\right)-\mu_{c} \sum_{r ; i=1}^{M} c_{r i}^{\dagger} c_{r i}-\mu \sum_{r ; i=1}^{N} f_{r i}^{\dagger} f_{r i} \\
& +\frac{1}{N M^{1 / 2}} \sum_{r ; i, j=1}^{N} \sum_{k, l=1}^{M} g_{i j k l}^{r} f_{r i}^{\dagger} f_{r j} c_{r k}^{\dagger} c_{r l} \\
& +\frac{1}{N^{3 / 2}} \sum_{r ; i, j, k, l=1}^{N} J_{i j k l}^{r} f_{r i}^{\dagger} f_{r j}^{\dagger} f_{r k} f_{r l} .
\end{aligned}
$$

We will take the limits of $M=\infty$ and $N=\infty$, but we will be interested in values of $M / N$ that are at most $\mathcal{O}(1)$. We choose $J_{i j k l}^{r}$ and $g_{i j k l}^{r}$ as independent complex Gaussian random variables, with $\left\langle\left\langle J_{i j k l}^{r} J_{l k i j}^{r^{\prime}}\right\rangle\right\rangle=\left(J^{2} / 8\right) \delta_{r r^{\prime}}$ and $\left\langle\left\langle g_{i j k l}^{r} g_{j i l k}^{r^{\prime}}\right\rangle\right\rangle=g^{2} \delta_{r r^{\prime}}$ and all other $\left\langle\left\langle{ }^{*}\right\rangle\right\rangle$ 's being zero, where $\langle\langle "\rangle\rangle$ denotes disorder averaging. Note that $t$ is nonrandom, and this will lead to a Fermi surface for the $c$ fermions. The disorder-averaged action then is 


$$
\begin{aligned}
S= & \int_{0}^{\beta} d \tau\left[\sum_{r ; i=1}^{M} c_{r i}^{\dagger}(\tau)\left(\partial_{\tau}-\mu_{c}\right) c_{r i}(\tau)-t \sum_{\left\langle r r^{\prime}\right\rangle ; i=1}^{M}\left(c_{r i}^{\dagger}(\tau) c_{r^{\prime} i}(\tau)+\text { H.c. }\right)+\sum_{r ; i=1}^{N} f_{r i}^{\dagger}(\tau)\left(\partial_{\tau}-\mu\right) f_{r i}\left(\tau^{\prime}\right)\right] \\
& -M \frac{g^{2}}{2} \sum_{r} \int_{0}^{\beta} d \tau d \tau^{\prime} G_{r}^{c}\left(\tau-\tau^{\prime}\right) G_{r}^{c}\left(\tau^{\prime}-\tau\right) G_{r}\left(\tau-\tau^{\prime}\right) G_{r}\left(\tau^{\prime}-\tau\right)-N \frac{J^{2}}{4} \sum_{r} \int_{0}^{\beta} d \tau d \tau^{\prime} G_{r}^{2}\left(\tau-\tau^{\prime}\right) G_{r}^{2}\left(\tau^{\prime}-\tau\right) \\
& -N \sum_{r} \int_{0}^{\beta} d \tau d \tau^{\prime} \Sigma_{r}\left(\tau-\tau^{\prime}\right)\left(G_{r}\left(\tau^{\prime}-\tau\right)+\frac{1}{N} \sum_{i=1}^{N} f_{r i}^{\dagger}(\tau) f_{r i}\left(\tau^{\prime}\right)\right) \\
& -M \sum_{r} \int_{0}^{\beta} d \tau d \tau^{\prime} \Sigma_{r}^{c}\left(\tau-\tau^{\prime}\right)\left(G_{r}^{c}\left(\tau^{\prime}-\tau\right)+\frac{1}{M} \sum_{i=1}^{M} c_{r i}^{\dagger}(\tau) c_{r i}\left(\tau^{\prime}\right)\right),
\end{aligned}
$$

where we have followed the usual strategy for SYK models $[23,28]$ and introduced the auxiliary fields $G, \Sigma, G^{c}$, and $\Sigma^{c}$, corresponding to Green's functions and self-energies of the $f$ and $c$ fermions, respectively, at each lattice site. In the $M$, $N=\infty$ limit, the integrals over the $\Sigma, \Sigma^{c}$ fields enforce the definitions of $G, G^{c}$ at each lattice site $r$. The large $M, N$ saddlepoint equations are obtained by varying the action with respect to these $G$ and $\Sigma$ fields after integrating out the fermions:

$$
\begin{aligned}
\Sigma_{r}\left(\tau-\tau^{\prime}\right) & =\Sigma\left(\tau-\tau^{\prime}\right)=-J^{2} G_{r}^{2}\left(\tau-\tau^{\prime}\right) G_{r}\left(\tau^{\prime}-\tau\right)-\frac{M}{N} g^{2} G_{r}\left(\tau-\tau^{\prime}\right) G_{r}^{c}\left(\tau-\tau^{\prime}\right) G_{r}^{c}\left(\tau^{\prime}-\tau\right) \\
& =-J^{2} G^{2}\left(\tau-\tau^{\prime}\right) G\left(\tau^{\prime}-\tau\right)-\frac{M}{N} g^{2} G\left(\tau-\tau^{\prime}\right) G^{c}\left(\tau-\tau^{\prime}\right) G^{c}\left(\tau^{\prime}-\tau\right), \\
G\left(i \omega_{n}\right) & =\frac{1}{i \omega_{n}+\mu-\Sigma\left(i \omega_{n}\right)},
\end{aligned}
$$

and

$$
\begin{aligned}
\Sigma_{r}^{c}\left(\tau-\tau^{\prime}\right) & =\Sigma^{c}\left(\tau-\tau^{\prime}\right)=-g^{2} G_{r}^{c}\left(\tau-\tau^{\prime}\right) G_{r}\left(\tau-\tau^{\prime}\right) G_{r}\left(\tau^{\prime}-\tau\right)=-g^{2} G^{c}\left(\tau-\tau^{\prime}\right) G\left(\tau-\tau^{\prime}\right) G\left(\tau^{\prime}-\tau\right), \\
G^{c}\left(i \omega_{n}\right) & =\int \frac{d^{d} k}{(2 \pi)^{d}} \frac{1}{i \omega_{n}-\epsilon_{k}+\mu_{c}-\Sigma^{c}\left(i \omega_{n}\right)} \equiv \int \frac{d^{d} k}{(2 \pi)^{d}} G^{c}\left(k, i \omega_{n}\right) .
\end{aligned}
$$

The last expression shows that the $c$ fermions have a dispersion $\epsilon_{k}$ and an associated Fermi surface; the lifetime of the Fermi surface excitations will be determined by the frequency dependence of $\Sigma^{c}$, which will be computed in the next section. We define chemical potentials such that halffilling occurs when $\mu=\mu_{c}=0$. The islands are not capable of exchanging electrons with the Fermi sea, so there is no reason a priori to have $\mu=\mu_{c}$, or even for islands at different sites to have the same $\mu$. However, for convenience, we will keep the $\mu$ of all the islands the same. The real system would operate at fixed densities, and $\mu$ and $\mu_{c}$ will appropriately renormalize as the mutual coupling $g$ is varied, in order to keep the densities of $c$ and $f$ individually fixed, as the interaction between $c$ and $f$ conserves their numbers individually. However, as we shall find, the halffilled case always corresponds to $\mu=\mu_{c}=0$ regardless of $g$. We will always have $J \gg T$ in this work, and also $J \gtrsim g$. A sketch of the phases realized by our model as a function of temperature is shown in Fig. 1(b).

\section{FATE OF THE CONDUCTION ELECTRONS}

\section{A. The case of infinite bandwidth}

We first consider the case of infinite bandwidth or, equivalently, $t \gg g, J \gg T$. The precise value of $\mu_{c}$ does not matter as long as its magnitude is not infinite, as the conduction electrons float on an effectively infinitely deep Fermi sea. Then, we can use the standard trick for evaluating integrals about a Fermi surface, and we have

$$
\begin{aligned}
G^{c}\left(i \omega_{n}\right) & =\int \frac{d^{d} k}{(2 \pi)^{d}} \frac{1}{i \omega_{n}-\epsilon_{k}+\mu_{c}-\Sigma^{c}\left(i \omega_{n}\right)} \\
& \rightarrow \nu(0) \int_{-\infty}^{\infty} \frac{d \varepsilon}{2 \pi} \frac{1}{i \omega_{n}-\varepsilon-\Sigma^{c}\left(i \omega_{n}\right)},
\end{aligned}
$$

where $\nu(0)$ is the density of states at the Fermi energy.

We take the lattice constant $a$ to be 1 . This makes $k$ dimensionless by redefining $k a$ to be $k$. The energy dimension of $\epsilon_{k}$ then comes from the inverse band mass. The density of states $\nu(0)$ then has the dimension of $1 /$ (energy) [on a lattice $\nu(0) \sim 1 / t \sim 1 / \Lambda$, where $\Lambda$ is the bandwidth].

We will also have $\operatorname{sgn}\left(\operatorname{Im}\left[\Sigma^{c}\left(i \omega_{n}\right)\right]\right)=-\operatorname{sgn}\left(\omega_{n}\right)$, so

$$
\begin{aligned}
G^{c}\left(i \omega_{n}\right) & =-\frac{i}{2} \nu(0) \operatorname{sgn}\left(\omega_{n}\right), \\
G^{c}(\tau) & =-\frac{\nu(0) T}{2 \sin (\pi T \tau)}, \quad-\beta \leq \tau \leq \beta,
\end{aligned}
$$


with other intervals obtained by applying the KuboMartin-Schwinger (KMS) condition $G^{c}(\tau+\beta)=-G^{c}(\tau)$. At $T=0$, we have

$$
G^{c}(\tau, T=0)=-\frac{\nu(0)}{2 \pi \tau} .
$$

We consider $M / N=0$ to begin with. Then, the $f$ electrons are not affected by the $c$ electrons, and their Green's functions are exactly of the incoherent form of the SYK model, which, in the low-energy limit, are given by [23]

$$
\begin{aligned}
G(\tau)= & -\frac{\pi^{1 / 4} \cosh ^{1 / 4}(2 \pi \mathcal{E})}{J^{1 / 2} \sqrt{1+e^{-4 \pi \mathcal{E}}}} \\
& \times\left(\frac{T}{\sin (\pi T \tau)}\right)^{1 / 2} e^{-2 \pi \mathcal{E} T \tau}, \quad 0 \leq \tau<\beta,
\end{aligned}
$$

where $\mathcal{E}$ is a function of $\mu$, with $\mathcal{E} \propto-\mu / J$ for small $\mu / J$. Other intervals are again obtained by the KMS condition $G(\tau+\beta)=-G(\tau)$. The zero-temperature limit of this, and similar expressions appearing later, can be straightforwardly taken [23]:

$$
\begin{aligned}
& G(\tau>0, T=0)=-\frac{\cosh ^{1 / 4}(2 \pi \mathcal{E})}{\pi^{1 / 4} J^{1 / 2} \sqrt{1+e^{-4 \pi \mathcal{E}}}} \frac{1}{\tau^{1 / 2}}, \\
& G(\tau<0, T=0)=\frac{\cosh ^{1 / 4}(2 \pi \mathcal{E})}{\pi^{1 / 4} J^{1 / 2} \sqrt{1+e^{4 \pi \mathcal{E}}}} \frac{1}{|\tau|^{1 / 2}}
\end{aligned}
$$

Now we can compute the self-energy of the $c$ fermions, which is

$$
\begin{aligned}
\Sigma^{c}(\tau) & =-g^{2} G^{c}(\tau) G(\tau) G(-\tau) \\
& =-\frac{\pi^{1 / 2} g^{2} \nu(0) T^{2}}{4 J \cosh ^{1 / 2}(2 \pi \mathcal{E}) \sin ^{2}(\pi T \tau)}, \quad 0 \leq \tau<\beta .
\end{aligned}
$$

Fourier transforming with a cutoff of $\tau$ at $J^{-1} \ll T^{-1}$ and $\beta-J^{-1}$ gives

$$
\begin{aligned}
\Sigma^{c}\left(i \omega_{n}\right)= & \frac{i g^{2} \nu(0) T}{2 J \cosh ^{1 / 2}(2 \pi \mathcal{E}) \pi^{3 / 2}} \\
& \times\left(\frac{\omega_{n}}{T} \ln \left(\frac{2 \pi T e^{\gamma_{E}-1}}{J}\right)+\frac{\omega_{n}}{T} \psi\left(\frac{\omega_{n}}{2 \pi T}\right)+\pi\right),
\end{aligned}
$$

where $\psi$ is the digamma function and $\gamma_{E}$ is the Euler-Mascheroni constant. As foreseen, this satisfies $\operatorname{sgn}\left(\operatorname{Im}\left[\Sigma^{c}\left(i \omega_{n}\right)\right]\right)=-\operatorname{sgn}\left(\omega_{n}\right)$ on the fermionic Matsubara frequencies. For $\left|\omega_{n}\right| \gg T$,

$$
\Sigma^{c}\left(i \omega_{n}\right) \rightarrow \frac{i g^{2} \nu(0)}{2 J \cosh ^{1 / 2}(2 \pi \mathcal{E}) \pi^{3 / 2}} \omega_{n} \ln \left(\frac{\left|\omega_{n}\right| e^{\gamma_{E}-1}}{J}\right) .
$$

Note the MFL form of the itinerant $c$ fermion self-energy, $\sim \omega \ln \omega$. Since the large $N$ and $M$ limits are taken at the outset, this MFL is stable even as $T \rightarrow 0$. For finite $N$ and $M$, the coupling $g$ is irrelevant in the infrared (IR) [33], and the model reduces to a theory of noninteracting electrons as $T \rightarrow 0$, with the MFL existing only above a temperature scale whose magnitude is suppressed by $N$ and the zerotemperature entropy going to zero.

Upon analytically continuing $i \omega_{n} \rightarrow \omega+i 0^{+}$, we get the inverse lifetime for the conduction electrons defined by

$$
\begin{aligned}
\gamma & \equiv-2 \operatorname{Im}\left[\Sigma_{R}^{c}(0)\right] \equiv-\operatorname{Im}\left[\Sigma^{c}\left(i \omega_{n} \rightarrow 0+i 0^{+}\right)\right] \\
& =\frac{g^{2} \nu(0) T}{J \cosh ^{1 / 2}(2 \pi \mathcal{E}) \pi^{1 / 2}} .
\end{aligned}
$$

Since the coupling of the conduction electrons to the SYK islands is spatially disordered, this rate also represents the transport scattering rate up to a constant numerical factor. The scattering of $c$ electrons off the islands requires the $f$ electrons inside the islands to move between orbitals. Hence, $\gamma$ vanishes when the islands are flooded or drained by sending $\mathcal{E} \rightarrow \mp \infty$, respectively, say, by doping them.

If we do not have $M / N=0$, the SYK Green's function will be affected, as there is a backreaction self-energy to the SYK islands. To see what this does when we perturbatively turn on $M / N$, we compute it with the $M / N=0$ Green's functions, with a cutoff of $\tau$ at $J^{-1}$ and $\beta-J^{-1}$ :

$$
\begin{aligned}
\tilde{\Sigma}(\tau) & =-\frac{M}{N} g^{2} G(\tau) G^{c}(\tau) G^{c}(-\tau) \\
& \approx-\frac{M \pi^{1 / 4} \cosh ^{1 / 4}(2 \pi \mathcal{E}) g^{2} \nu^{2}(0) T^{5 / 2} e^{-2 \pi \mathcal{E} T \tau}}{4 N J^{1 / 2} \sqrt{1+e^{-4 \pi \mathcal{E}}} \sin ^{5 / 2}(\pi T \tau)} .
\end{aligned}
$$

If $\mathcal{E}=0$, then $\tilde{\Sigma}\left(i \omega_{n}\right) \propto i(M / N) g^{2} \nu^{2}(0) \omega_{n}$ as $T, \omega_{n} \rightarrow 0$, which is subleading to $\left.\Sigma\left(i \omega_{n}\right)\right|_{M / N=0} \sim\left(J \omega_{n}\right)^{1 / 2}$, so the SYK character of the islands survives in the IR.

Now we consider the case of particle-hole symmetry breaking with a nonzero spectral asymmetry, $\mathcal{E}$ in Eq. (1.1); we will find that the basic structure of the results described above persists. If $\mathcal{E} \neq 0$ but is small, then for $T \rightarrow 0$, $\tilde{\Sigma}\left(i \omega_{n} \rightarrow 0\right) \sim-(M / N) g^{2} \nu^{2}(0) J \mathcal{E} \propto(M / N) g^{2} \nu^{2}(0) \mu+$ $\mathcal{O}\left(i \omega_{n}\right)$. In contrast, $\left.\Sigma\left(i \omega_{n} \rightarrow 0\right)\right|_{M / N=0} \sim \mu+\mathcal{O}\left(\omega_{n}^{1 / 2}\right)$. Therefore, the frequency-dependent part of $\tilde{\Sigma}$ is still subleading. Hence, in the IR we may still assume that all that happens to the SYK islands is that their chemical potential $\mu$ gets renormalized. By solving $\operatorname{Re}\left[\Sigma\left(i \omega_{n} \rightarrow 0, T=0\right)\right]=\mu$, we obtain the corrected $\mathcal{E} \leftrightarrow \mu$ relation. At small $\mu / J$, this is

$$
\mathcal{E} \approx-\frac{\mu / J}{\pi^{1 / 4} \sqrt{2}\left(1+\frac{g^{2} \nu^{2}(0) M}{6 \pi^{3 / 2} N}\right)} .
$$


The total particle number on each island, $\mathcal{N}_{r}=\sum_{i} f_{i r}^{\dagger} f_{i r}$, commutes with $H$. Since the SYK particle density $\mathcal{Q}=$ $\mathcal{N} / N$ is a universal function of $\mathcal{E}$, independent of $\mu$ and $J$, Eq. (3.11) just implies a renormalization of the nonuniversal UV parts of the SYK Green's function and the island chemical potential, while the particle density remains fixed. Similarly, the vanishing of the zero-frequency real part of Eq. (3.7) regardless of $\mathcal{E}$ implies that there is no renormalization of either the density or chemical potential of the conduction electrons in this infinite-bandwidth limit, since their number is independently conserved as well. For a finite bandwidth, the chemical potential of the conduction electrons renormalizes in such a way that their density remains fixed.

In Appendix A, we consider the effects of adding a "pairhopping" term to Eq. (2.1),

$H \rightarrow H+\frac{1}{N M^{1 / 2}} \sum_{r, i, j=1}^{N} \sum_{k, l=1}^{M}\left[\eta_{i j k l}^{r} f_{r i}^{\dagger} f_{r j}^{\dagger} c_{r k} c_{r l}+\right.$ H.c. $]$,

with $\left\langle\left\langle\left|\eta_{i j k l}^{r}\right|^{2}\right\rangle\right\rangle=\eta^{2} / 8$, and $J \gtrsim \eta$. This term has identical power-counting to the $f^{\dagger} f c^{\dagger} c$ term, but can trade $c$ electrons for $f$ electrons and vice versa. Since the numbers of $c$ and $f$ electrons are no longer independently conserved in this case, there is only one chemical potential, and $\mu_{c}=\mu$. We find that this term also generates a MFL as long as the bandwidth of the $c$ electrons is large.

As is well known, the marginal-Fermi liquid selfenergy we obtained in Eqs. (3.7) and (3.8) also leads to the leading low-temperature contribution to the specific heat coming from the itinerant electrons scaling as $C_{V}^{\mathrm{MFL}} \sim$ $M g^{2}[\nu(0)]^{2}(T / J) \ln (J / T)$ [41]. Note that the entropy has a nonvanishing $T \rightarrow 0$ limit from the contribution of the SYK islands in the limit of $N \rightarrow \infty$ [42], but this does not contribute to the specific heat. The contribution to the specific heat coming from the SYK islands scales linearly in $T$ as $T \rightarrow 0$ [28], which is subleading to the $T \ln T$ contribution of the itinerant electrons.

\section{B. The case of a finite bandwidth}

This subsection will show that a finite bandwidth does not modify the basic structure of the low-temperature MFL phase described above. However, if interactions between $c$ and $f$ are strong enough, a crossover into an IM phase is possible at higher temperatures. Readers not interested in the details of the arguments can move ahead to the next section.

If the bandwidth (and hence Fermi energy) of the conduction electrons is sizable compared to the couplings, then the momentum-integrated local Green's function $G^{c}\left(i \omega_{n}\right)$ is no longer independent of the details of the self-energy $\Sigma^{c}\left(i \omega_{n}\right)$. We consider two spatial dimensions, with the isotropic dispersion $\varepsilon_{k}=k^{2} /(2 m)-\Lambda / 2$, and a bandwidth $\varepsilon_{k}^{\max }-\varepsilon_{k=0}=\Lambda$. Since $k$ is dimensionless, the band mass $m$ has dimensions of $1 /$ (energy). The density of states is then just $\nu(\varepsilon)=\nu(0)=m$, at all energies $\varepsilon$, and we implicitly make use of this fact while simplifying and rewriting certain expressions. On a lattice, $m \sim \nu(0) \sim$ $1 / t \sim 1 / \Lambda$.

The momentum-integrated conduction electron Green's function is

$$
\begin{aligned}
G^{c}\left(i \omega_{n}\right)= & \frac{\nu(0)}{2 \pi}\left(\ln \left[\Lambda+2 \mu_{c}+2 i \omega_{n}-2 \Sigma^{c}\left(i \omega_{n}\right)\right]\right. \\
& \left.-\ln \left[2 \mu_{c}-\Lambda+2 i \omega_{n}-2 \Sigma^{c}\left(i \omega_{n}\right)\right]\right) .
\end{aligned}
$$

We still expect $\operatorname{sgn}\left(\operatorname{Im}\left[\Sigma^{c}\left(i \omega_{n}\right)\right]\right)=-\operatorname{sgn}\left(\omega_{n}\right)$. The chemical potential $\mu_{c}$ must now take an appropriate value to reproduce the correct density of conduction electrons. The conduction band filling is given by

$$
\mathcal{Q}_{c}=\frac{2 \pi G^{c}\left(\tau=0^{-}\right)}{\nu(0) \Lambda},
$$

for the exact solution to $G^{c}$, which can be found by the imaginary-time MATLAB code gg c . m [43] (the low-energy "conformal-limit" solutions described below are not valid at the short times $0^{-}$and do not display this property).

In general, the Dyson equations can now only be solved numerically, which the imaginary-time MATLAB code ggc.m [43] and real-time MATLAB code ggcrealtime.m [44] do, albeit by holding the chemical potentials $\mu$ and $\mu_{c}$, rather than densities, fixed. In an extreme limit where $\mid i \omega_{n}+$ $\mu_{c}-\Sigma^{c}\left(i \omega_{n}\right) \mid$ far exceeds the bandwidth for all $\omega_{n}$, which can happen only at $T \neq 0$, we have a simplification of Eq. (3.13), obtained by expanding in $\Lambda$,

$$
G^{c}\left(i \omega_{n}\right)=\frac{\Lambda \nu(0)}{2 \pi\left[i \omega_{n}+\mu_{c}-\Sigma^{c}\left(i \omega_{n}\right)\right]} .
$$

This then leads to a SYK solution in the low-energy conformal limit for both $G$ and $G^{c}$, realizing a fully incoherent metal. We use the trial solutions

$$
\begin{aligned}
G^{c}(\tau) & =-\frac{C_{c}}{\sqrt{1+e^{-4 \pi \mathcal{E}_{c}}}}\left(\frac{T}{\sin (\pi T \tau)}\right)^{1 / 2} e^{-2 \pi \mathcal{E}_{c} T \tau}, \\
G(\tau) & =-\frac{C}{\sqrt{1+e^{-4 \pi \mathcal{E}}}}\left(\frac{T}{\sin (\pi T \tau)}\right)^{1 / 2} e^{-2 \pi \mathcal{E} T \tau}, \quad 0 \leq \tau<\beta .
\end{aligned}
$$

$\mathcal{E}_{c}$ is universally related to the conduction band filling, with $\mathcal{E}_{c}=0$ at half filling, and $\mathcal{E}_{c} \rightarrow \mp \infty$ when the band is full or empty, respectively. When $M / N=0$, there is no backreaction to the islands, and $G$ is given by (3.4). We use the conditions $\operatorname{Re}\left[\Sigma^{c}\left(i \omega_{n} \rightarrow 0, T=0\right)\right]=\mu_{c}$ and $G^{c}\left(i \omega_{n} \rightarrow 0, T=0\right)=\Lambda \nu(0) /\left(2 \pi\left[\mu_{c}-\Sigma^{c}\left(i \omega_{n} \rightarrow 0, T=0\right)\right]\right)$ to determine $C_{c}$, and also $\mu_{c}$ in terms of the fixed $\mathcal{E}_{c}$. Cutting 
off $\tau$ integrals in the Fourier transforms at a distance $\alpha_{\mathrm{UV}}^{-1}$ from singularities, we have

$$
\begin{aligned}
& C_{c}=\frac{\cosh ^{1 / 4}(2 \pi \mathcal{E})}{2^{1 / 2} \pi^{1 / 4} J_{\mathrm{IM}}^{1 / 2}}, \quad J_{\mathrm{IM}} \equiv \frac{g^{2}}{J \Lambda \nu(0)}, \text { and } \\
& \mathcal{E}_{c} \approx-\frac{\pi^{1 / 4} \cosh ^{1 / 4}(2 \pi \mathcal{E}) \mu_{c}}{g \Lambda^{1 / 2} \nu^{1 / 2}(0)} \sqrt{\frac{J}{\alpha_{\mathrm{UV}}}}\left(\text { at small } \mu_{c} / g\right),
\end{aligned}
$$

with no feedback on the SYK islands. For Eq. (3.15) to derive from Eq. (3.13), this requires $\left|\mu_{c}-\Sigma^{c}\left(i \omega_{n} \rightarrow 0\right)\right| \gg \Lambda$ or

$$
T \gg T_{\mathrm{inc}} \equiv \frac{\Lambda J}{\nu(0) g^{2}} .
$$

Furthermore, for Eqs. (3.4) and (3.16) to hold, we also need $J \gg T_{\text {inc }}$ and $J_{\text {IM }} \gg T_{\text {inc }}$, implying $g^{2} \gg \Lambda J$. For $T \ll T_{\text {inc }}$, we go back to the MFL, which now has a UV cutoff of $T_{\text {inc }}$ instead of $J$, with its self-energy going as $\Sigma^{c}\left(i \omega_{n}\right) \sim\left(g^{2} \nu(0) / J\right) i \omega_{n} \ln \left(\left|\omega_{n}\right| / T_{\text {inc }}\right)$. The choice of the UV cutoff $\alpha_{\mathrm{UV}}$ in the IM only affects the nonuniversal $\mathcal{E}_{c} \leftrightarrow \mu_{c}$ relation. An appropriate choice of the cutoff is $\alpha_{\mathrm{UV}} \sim J_{\mathrm{IM}} \lesssim J$.

Turning on a small but finite $M / N$, we have to additionally use the conditions $\operatorname{Re}\left[\Sigma\left(i \omega_{n} \rightarrow 0, T=0\right)\right]=\mu$ and $G\left(i \omega_{n} \rightarrow 0, T=0\right)=1 /\left[\mu-\Sigma\left(i \omega_{n} \rightarrow 0, T=0\right)\right]$ simultaneously to determine a renormalized $C$ and renormalized $\mu$, while keeping $\mathcal{E}$ fixed as before. We again cut off $\tau$ integrals in the Fourier transforms at a distance $\alpha_{\mathrm{UV}}^{-1}$ from singularities. This gives

$$
\begin{aligned}
C & =\cosh ^{1 / 4}(2 \pi \mathcal{E}) \frac{\pi^{1 / 4}}{J^{1 / 2}}\left(1-\frac{M}{N} \frac{\Lambda \nu(0)}{2 \pi} \frac{\cosh (2 \pi \mathcal{E})}{\cosh \left(2 \pi \mathcal{E}_{c}\right)}\right)^{1 / 4}, \\
C_{c} & =\frac{\cosh ^{1 / 2}(2 \pi \mathcal{E}) \Lambda^{1 / 2} \nu^{1 / 2}(0)}{2^{1 / 2} C g}
\end{aligned}
$$

and we do not show the nonuniversal $\mathcal{E}, \mathcal{E}_{c} \leftrightarrow \mu, \mu_{c}$ relations because they are rather uninsightful and the physics is better described in terms of $\mathcal{E}, \mathcal{E}_{c}$, which universally represent the conserved densities.

If $M / N$ is increased to approach $\left[2 \pi \cosh \left(2 \pi \mathcal{E}_{c}\right)\right] /$ $[\Lambda \nu(0) \cosh (2 \pi \mathcal{E})]$, the condition for incoherence that $\left|i \omega_{n}+\mu_{c}-\Sigma^{c}\left(i \omega_{n}\right)\right|$ exceed the bandwidth for all $\omega_{n}$ becomes harder to fulfill, and larger and larger values of the coupling $g$ are required to achieve the IM phase at high temperatures.

When $M / N>\left[2 \pi \cosh \left(2 \pi \mathcal{E}_{c}\right)\right] /[\Lambda \nu(0) \cosh (2 \pi \mathcal{E})]$, we still recover the MFL deep enough in the IR, due to the backreaction self-energy $\tilde{\Sigma}$ being irrelevant, and the conduction electron self-energy $\Sigma^{c}$ also vanishing at the lowest energies. However, at values of the coupling $g$ large enough so that effects of the conduction electron bandwidth may be ignored above a certain temperature, we find a crossover into a different IM phase, with local Green's functions given by (at half-filling)

$$
\begin{aligned}
G^{c}(\tau) & \sim\left(\frac{T}{\sin (\pi T \tau)}\right)^{\Delta_{c}}, \\
G(\tau) & \sim\left(\frac{T}{\sin (\pi T \tau)}\right)^{1-\Delta_{c}}, \quad 0<\Delta_{c}<1 / 2
\end{aligned}
$$

with $\Delta_{c}$ given by the solution to the equation

$$
\left(\frac{\Delta_{c}}{1-\Delta_{c}}\right) \cot ^{2}\left(\frac{\pi \Delta_{c}}{2}\right)=\frac{M}{N} \frac{\Lambda \nu(0)}{2 \pi},
$$

which has the property that $\Delta_{c} \rightarrow 0$ as $M / N \rightarrow \infty$ and $\Delta_{c} \rightarrow 1 / 2$ as $M / N \rightarrow 2 \pi /[\Lambda \nu(0)]$. These Green's functions may be derived by solving the Dyson equations (2.3) and (2.4), while ignoring both the conduction electron dispersion and the coupling $J$. Indeed, with the scalings in Eq. (3.20), the term proportional to $J^{2}$ in the expression for $\Sigma(\tau)$ is irrelevant compared to the other term. This phase has a resistivity that scales as $T^{2\left(1-\Delta_{c}\right)}$. Since we are only interested in models with linear-in- $T$ resistivities, we will henceforth assume that $M / N$ is small enough to avoid this regime.

Since $\nu(0) \sim 1 / \Lambda \sim 1 / t$ on a lattice, fine-tuning $g \sim J \sim$ $\Lambda \gg T$ makes the scattering rate in Eq. (3.9) "Planckian," i.e., an $\mathcal{O}(1)$ number times $T$, since it is given by ratios of large quantities. The MFL does not break down if we do this. In Eq. (3.13), $\left|\Sigma^{c}\left[i\left(\omega_{n} \sim T\right)\right]\right| \sim T \ln T / J \ll \Lambda$, so the infinite-bandwidth result Eq. (3.9) is still applicable. The crossover to the IM does not occur either, since $T \ll T_{\text {inc }}$, and finally, the part of the backreaction self-energy to the SYK islands that does not renormalize their chemical potentials is $\left|\tilde{\Sigma}\left[i\left(\omega_{n} \sim T\right)\right]\right| \sim(M / N)[g \nu(0)]^{2} T$, which is $\ll\left|\Sigma\left[i\left(\omega_{n} \sim T\right)\right]\right| \sim(J T)^{1 / 2}$, i.e., the part of the internal self-energy of the SYK islands that does not renormalize the chemical potential, as long as $M / N$ is not $\gg 1$, so the SYK character of the islands also survives.

In the IM regime, since both the conduction and islands electrons have local SYK Green's functions, the specific heat scales as $C_{V}^{\mathrm{IM}} \sim M T / J_{\mathrm{IM}}+N T / J$, with no logarithmic corrections [28].

\section{TRANSPORT IN A SINGLE DOMAIN}

In this section, we consider transport in two spatial dimensions, with the isotropic dispersion $\varepsilon_{k}=k^{2} /(2 m)-$ $\Lambda / 2$. We will find that many aspects of the transport can be computed in a traditional Boltzmann transport computation, due to the large $N$ and $M$ limits. In particular, quantum corrections to transport, of the type leading to quantum interference and localization, are suppressed by the local disorder, the nonquasiparticle nature of the charge carriers, and the large number of fermion flavors. 
(a)



FIG. 2. (a) The uniform current-current correlation bubble used to compute conductivities. The current vertices are black squares and the black lines are conduction electron (c) propagators. (b), (c) Additional diagrams forming ladder series, with ladder units of up to three loops, that contribute to the conductivities and are not immediately suppressed by the large $N$ and $M$ limits. The red lines are island fermion $(f)$ propagators that do not carry momentum. The dashed blue lines carry momentum and come from disorder averaging of the nontranslationally invariant coupling $g_{i j k l}^{x}$. These diagrams, however, vanish upon momentum integration in the loops containing the current vertices, for reasons mentioned in the main text.

In our double large $N$ and $M$ limit, if $M / N=0$, the only vertex corrections to the uniform conductivities that are not trivially killed by this limit are the ones that involve uncrossed vertical ladders of $f_{i}^{\dagger} f_{j}$ propagators in the current-current correlator bubbles [first diagram of Fig. 2(b)]. However, since the $f$ propagators are purely local and independent of momentum, these diagrams vanish due to the averaging of the vector velocity in the current vertices over the closed fixed-energy contours in momentum space, as the scattering of the conduction electrons is isotropic, just like in the textbook problem of the noninteracting disordered metal [45]. Unlike the noninteracting disordered metal, there is no localization in two dimensions as the crossed-ladder "Cooperon" diagrams are suppressed by the large $M$ limit. Hence, the relaxation-time-like approximation of keeping only self-energy corrections is valid.

If $M / N$ is nonzero but $\mathcal{O}(1)$ or smaller, then certain three-loop and higher order ladder insertions [such as Fig. 2(c)] also contribute extensively in $M$ to the current-current correlation. However, these diagrams again vanish due to the averaging of the vector velocity mentioned above. All of this happens regardless of the values of $g, J, \Lambda$, or $\mu_{c}$, and for both energy and electrical currents.

\section{A. Marginal-Fermi liquid}

We first discuss a Boltzmann transport approach in the MFL regime. For simplicity, we consider infinite bandwidth and an infinitely deep Fermi sea. The uniform current-current correlation bubble [Fig. 2(a)] is given by, for an isotropic Fermi surface,

$$
\left\langle I_{x} I_{x}\right\rangle\left(i \Omega_{m}\right)=-M \frac{v_{F}^{2}}{2} \nu(0) T \sum_{\omega_{n}} \int_{-\infty}^{\infty} \frac{d \varepsilon}{2 \pi} \frac{1}{i \omega_{n}-\varepsilon-\Sigma^{c}\left(i \omega_{n}\right)} \frac{1}{i \omega_{n}+i \Omega_{m}-\varepsilon-\Sigma^{c}\left(i \omega_{n}+i \Omega_{m}\right)},
$$

where $v_{F}=k_{F} / m$ is the Fermi velocity (on a lattice $v_{F} \sim t$, since the lattice constant $a$ is set to 1). Using the spectral representation, this can be converted to give the DC conductivity

$\sigma_{0}^{\mathrm{MFL}}=M \frac{v_{F}^{2} \nu(0)}{16 T} \int_{-\infty}^{\infty} \frac{d E_{1}}{2 \pi} \operatorname{sech}^{2}\left(\frac{E_{1}}{2 T}\right) \frac{1}{\left|\operatorname{Im} \Sigma_{R}^{c}\left(E_{1}\right)\right|}$.

Inserting the self-energy, we can scale out $T$ and numerically evaluate the integral, giving

$\sigma_{0}^{\mathrm{MFL}}=0.120251 \times M T^{-1} J \times\left(\frac{v_{F}^{2}}{g^{2}}\right) \cosh ^{1 / 2}(2 \pi \mathcal{E})$.

If we want $\sigma_{0}^{\mathrm{MFL}} / M \ll 1$, we must have $T \gg T_{\mathrm{inc}}$, implying a crossover into the IM regime. Thus, the MFL is never a true bad metal, but its resistivity can still numerically exceed the quantum unit $h / e^{2}$, depending on parameters.

The "open-circuit" thermal conductivity $\kappa_{0}^{\mathrm{MFL}}$, which is defined under conditions where no electrical current flows, is given by

$$
\kappa_{0}^{\mathrm{MFL}}=\bar{\kappa}_{0}^{\mathrm{MFL}}-\frac{\left(\alpha_{0}^{\mathrm{MFL}}\right)^{2} T}{\sigma_{0}^{\mathrm{MFL}}},
$$

where $\bar{\kappa}_{0}^{\mathrm{MFL}}$ is the "closed-circuit" thermal conductivity in the presence of electrical current, and $\alpha_{0}^{\mathrm{MFL}}$ is the thermoelectric conductivity. The thermoelectric conductivity vanishes when the temperature is much smaller than the bandwidth and Fermi energy, due to effective particle-hole symmetry about the Fermi surface, so $\kappa_{0}^{\mathrm{MFL}}=\bar{\kappa}_{0}^{\mathrm{MFL}}$. The Lorenz ratio is then given by

$$
\begin{aligned}
L^{\mathrm{MFL}} & =\frac{\kappa_{0}^{\mathrm{MFL}}}{\sigma_{0}^{\mathrm{MFL}} T}=\frac{\bar{\kappa}_{0}^{\mathrm{MFL}}}{\sigma_{0}^{\mathrm{MFL}} T} \\
& =\frac{\int_{-\infty}^{\infty} \frac{d E_{1}}{2 \pi} E_{1}^{2} \operatorname{sech}^{2}\left(\frac{E_{1}}{2}\right) \frac{1}{\left|\operatorname{Im}\left(E_{1} \psi\left[-i E_{1} /(2 \pi)\right]+i \pi\right)\right|}}{\int_{-\infty}^{\infty} \frac{d E_{1}}{2 \pi} \operatorname{sech}^{2}\left(\frac{E_{1}}{2}\right) \frac{1}{\left|\operatorname{Im}\left(E_{1} \psi\left[-i E_{1} /(2 \pi)\right]+i \pi\right)\right|}} \\
& =0.713063 \times L_{0},
\end{aligned}
$$

which is smaller than $L_{0}=\pi^{2} / 3$ for a Fermi liquid.

In the presence of a uniform transverse magnetic field, we can use the following improved relaxation-time 
linearized Boltzmann equation (which incorporates an offshell distribution function) for a temporally slowly varying and spatially uniform applied electric field [46,47], since there are no Cooperons in the large- $M$ limit and, hence, none of the typical localization-related corrections [48] to the conductivity tensor. The Boltzmann equation reads (here, $t$ is time, not the hopping amplitude, and $\mathcal{B}$ is a dimensionless version of the magnetic field $B$, which shall be explained below)

$$
\begin{aligned}
& \left(1-\partial_{\omega} \operatorname{Re}\left[\Sigma_{R}^{c}(\omega)\right]\right) \partial_{t} \delta n(t, k, \omega)+v_{F} \hat{k} \cdot \mathbf{E}(t) n_{f}^{\prime}(\omega) \\
& +v_{F}(\hat{k} \times \mathcal{B} \hat{z}) \cdot \nabla_{k} \delta n(t, k, \omega) \\
& \quad=2 \delta n(t, k, \omega) \operatorname{Im}\left[\Sigma_{R}^{c}(\omega)\right]
\end{aligned}
$$

where $n_{f}(\omega)=1 /\left(e^{\omega / T}+1\right)$ is the Fermi distribution, $\delta n$ is the change in the distribution due to the applied electric field, the conduction electrons are negatively charged, and the magnetic field points out of the plane of the system. This equation is derived in Appendix B from the Dyson equation on the Keldysh contour and can be solved by the ansatz $\delta n(t, k, \omega)=k \cdot \varphi(t, \omega)=k_{i} \varphi_{i}(t, \omega)$.

In the DC limit, the effective mass enhancement $\left(1-\partial_{\omega} \operatorname{Re}\left[\Sigma^{R}(\omega)\right]\right)$ does not matter [47]. (The effective mass enhancement is important for AC magnetotransport and affects the frequency at which the cyclotron resonance occurs; it shifts the cyclotron resonance from the cyclotron frequency defined by the bare mass to the one defined by the effective mass. The enhanced effective mass also appears in the specific heat [41] and Lifshitz-Kosevich formula [49] of MFLs.) We then have

$$
\begin{aligned}
& v_{F} \hat{k} \cdot \mathbf{E} n_{f}^{\prime}(\omega)+v_{F}(\hat{k} \times \mathcal{B} \hat{z}) \cdot \nabla_{k} \delta n(k, \omega) \\
& \quad=2 \delta n(k, \omega) \operatorname{Im}\left[\Sigma_{R}^{c}(\omega)\right],
\end{aligned}
$$

We note that in Eq. (4.7), $\mathcal{B}$ is dimensionless in our choice of units. Since the quantities we set to 1 were the magnitude of the electron charge $e$, the lattice constant $a$, and $\hbar$ and $k_{B}$, we have

$$
\mathcal{B}=\frac{e B a^{2}}{\hbar}
$$

i.e., the flux per unit cell in units of $\hbar / e$.

Substituting $\delta n(k, \omega)=k_{i} \varphi_{i}(\omega)$ into Eq. (4.7), we obtain

$$
\varphi_{i}(\omega)=\frac{v_{F}}{k_{F}} n_{f}^{\prime}(\omega)\left(2 \operatorname{Im}\left[\Sigma_{R}^{c}(\omega)\right] \delta_{i j}+\epsilon_{i j} \mathcal{B} \frac{v_{F}}{k_{F}}\right)_{i j}^{-1} E_{j} .
$$

Using the current density,

$$
I_{i}=-M \nu(0) \int_{0}^{2 \pi} \frac{d \theta}{2 \pi} \int_{-\infty}^{\infty} \frac{d \omega}{2 \pi} v_{F} \hat{k}_{i} \delta n\left(k_{F} \hat{k}, \omega\right)
$$

we get the longitudinal and Hall conductivities

$$
\begin{aligned}
& \sigma_{L}^{\mathrm{MFL}}=M \frac{v_{F}^{2} \nu(0)}{16 T} \int_{-\infty}^{\infty} \frac{d E_{1}}{2 \pi} \operatorname{sech}^{2}\left(\frac{E_{1}}{2 T}\right) \frac{-\operatorname{Im}\left[\Sigma_{R}^{c}\left(E_{1}\right)\right]}{\operatorname{Im}\left[\Sigma_{R}^{c}\left(E_{1}\right)\right]^{2}+\left[v_{F} /\left(2 k_{F}\right)\right]^{2} \mathcal{B}^{2}}, \\
& \sigma_{H}^{\mathrm{MFL}}=-M \frac{v_{F}^{2} \nu(0)}{16 T} \int_{-\infty}^{\infty} \frac{d E_{1}}{2 \pi} \operatorname{sech}^{2}\left(\frac{E_{1}}{2 T}\right) \frac{\left(v_{F} /\left(2 k_{F}\right)\right) \mathcal{B}}{\operatorname{Im}\left[\Sigma_{R}^{c}\left(E_{1}\right)\right]^{2}+\left[v_{F} /\left(2 k_{F}\right)\right]^{2} \mathcal{B}^{2}} .
\end{aligned}
$$

Note that, given the scaling of Eq. (3.7), these can be immediately written as

$$
\begin{aligned}
\sigma_{L}^{\mathrm{MFL}} & \sim T^{-1} s_{L}\left[\left(v_{F} / k_{F}\right)(\mathcal{B} / T)\right], \\
\sigma_{H}^{\mathrm{MFL}} & \sim-\mathcal{B} T^{-2} s_{H}\left[\left(v_{F} / k_{F}\right)(\mathcal{B} / T)\right] .
\end{aligned}
$$

The asymptotic forms of the functions $s_{L}$ and $s_{H}$ are

$$
s_{L, H}(x \rightarrow \infty) \propto 1 / x^{2}, \quad s_{L, H}(x \rightarrow 0) \propto x^{0} .
$$

So, we have obtained the advertised $B / T$ scaling in the MFL regime. However, with the asymptotic forms noted above, it is not difficult to see that the magnetoresistance $\rho_{x x}$ saturates at large $B$. Nevertheless, the results above will be useful as inputs into our consideration of the effects of macroscopic disorder in Sec. V: We will show there that the
$B / T$ scaling survives, and the macroscopic disorder leads to a linear-in- $B$ magnetoresistance.

We now show that the numerical scale of the $B / T$ crossover is in general accord with the observations. In Eq. (4.11), for the "Planckian" choice of parameters described at the end of Sec. III B, B becomes "large" [i.e., the cyclotron term in the denominators overwhelms $\operatorname{Im}\left[\Sigma_{R}^{c}\left(E_{1}\right)\right]$ for $\left|E_{1}\right| \lesssim T$, causing $\sigma_{H}^{\mathrm{MFL}}$ to start decreasing with increasing $B]$, when $e B a^{2} / \hbar \gtrsim k_{B} T / t$. Using reasonable values of the lattice constant $a=3.82 \AA$ and the hopping $t=0.25 \mathrm{eV}$, the above inequality can also roughly be written as $\mu_{B} B \gtrsim k_{B} T$, where $\mu_{B}$ is the Bohr magneton, since $a^{2} e t / \hbar \approx 0.96 \mu_{B}$ for these parameters.

In the analysis of the IM regime to follow, there is no such notion of large magnetic fields; regardless of the value of $B$, the field-dependent corrections to the conductivity tensor remain much smaller than its zero-field value. 


\section{B. Incoherent metal}

This subsection considers transport in the IM phase discussed earlier, in which the Fermi surface is washed out, and shows quantitatively that the orbital effects of a magnetic field on charge transport are strongly suppressed irrespective of the strength of the field. The physical reason for this effect is that the effective mean-free path of the electrons in the IM is less than a lattice spacing, with conduction occurring locally and incoherently across individual lattice bonds. The effect of the Lorentz force on the electrons is, thus, negligible. If the reader is uninterested in the details of the following computations, they may move on to the next section.
In the IM regime, we have

$$
\sigma_{0}^{\mathrm{IM}}=\frac{M \Lambda^{2}}{32 \pi T} \int_{-\infty}^{\infty} \frac{d E_{1}}{2 \pi} \operatorname{sech}^{2}\left(\frac{E_{1}}{2 T}\right)\left[A^{c}\left(k, E_{1}\right)\right]^{2}
$$

The spectral function is independent of $k$ in the IM, and we decoupled the momentum integral implicit in the above equation, generating a prefactor of $\Lambda \nu(0) /(2 \pi)$. For simplicity, we consider $M / N=0$ in this subsection. A small finite $M / N$ only rescales $G^{c}$, as shown by Eqs. (3.19) and (3.16) and, hence, leads to no qualitative difference in any of the following results. We have

$$
\begin{aligned}
A^{c}\left(k, E_{1}\right) & \equiv \frac{2 \pi}{\Lambda \nu(0)} A^{c}\left(E_{1}\right) \equiv-\frac{4 \pi}{\Lambda \nu(0)} \operatorname{Im}\left[G^{c}\left(i \omega_{n} \rightarrow E_{1}+i 0^{+}\right)\right] \\
& =-2 \operatorname{Im}\left[\frac{i(-1)^{3 / 4} \pi^{1 / 4}\left(i+e^{2 \pi \mathcal{E}_{c}}\right) J^{1 / 2} \cosh ^{1 / 4}(2 \pi \mathcal{E})}{g T^{1 / 2} \Lambda^{1 / 2} \nu^{1 / 2}(0) \sqrt{1+e^{4 \pi \mathcal{E}_{c}}}} \frac{\Gamma\left(\frac{1}{4}-\frac{i\left(E_{1}-2 \pi \mathcal{E}_{c} T\right)}{2 \pi T}\right)}{\Gamma\left(\frac{3}{4}-\frac{i\left(E_{1}-2 \pi \mathcal{E}_{c} T\right)}{2 \pi T}\right)}\right],
\end{aligned}
$$

and we get

$$
\sigma_{0}^{\mathrm{IM}}=\left(\pi^{1 / 2} / 8\right) \times M T^{-1} J \times\left(\frac{\Lambda}{\nu(0) g^{2}}\right) \frac{\cosh ^{1 / 2}(2 \pi \mathcal{E})}{\cosh \left(2 \pi \mathcal{E}_{c}\right)} .
$$

Because the IM exists only at temperatures above $T_{\text {inc }}$, given by Eq. (3.18), we always have $\sigma_{0}^{\mathrm{IM}} / M \ll 1$, which makes the IM a bad metal. Note that the slope of the resistivity $\rho_{0}(T)=1 / \sigma_{0}(T)$ vs temperature in the IM generically differs from that in the MFL by an $\mathcal{O}(1)$ number, as can be seen by comparing Eqs. (4.3) and (4.16).

The Lorenz ratio in the IM is (here, the thermoelectric conductivity $\alpha_{0}^{\mathrm{IM}}$ does not vanish, so $\kappa_{0}^{\mathrm{IM}}$ and $\bar{\kappa}_{0}^{\mathrm{IM}}$ are distinct quantities)

$$
L^{\mathrm{IM}}=\frac{\int_{-\infty}^{\infty} \frac{d E_{1}}{2 \pi} E_{1}^{2} \operatorname{sech}^{2}\left(\frac{E_{1}}{2}\right)\left[A^{c}\left(E_{1}\right)\right]^{2}-\frac{\left[\int_{-\infty}^{\infty} \frac{d E_{1}}{2 \pi} E_{1} \operatorname{sech}^{2}\left(\frac{E_{1}}{2}\right)\left[A^{c}\left(E_{1}\right)\right]^{2}\right]^{2}}{\int_{-\infty}^{\infty} \frac{d E_{1}}{2 \pi} \operatorname{sech}^{2}\left(\frac{E_{1}}{2}\right)\left[A^{c}\left(E_{1}\right)\right]^{2}}}{\int_{-\infty}^{\infty} \frac{d E_{1}}{2 \pi} \operatorname{sech}^{2}\left(\frac{E_{1}}{2}\right)\left[A^{c}\left(E_{1}\right)\right]^{2}}=\frac{3}{8} \times L_{0}, \quad \text { regardless of } \mathcal{E}, \mathcal{E}_{c} .
$$

This result was also obtained by a different method for the IM of Ref. [10], although they only analyzed the particlehole symmetric case equivalent to $\mathcal{E}_{c}=0$.

Another dimensionless ratio that is interesting is the thermopower, i.e., the ratio of the thermoelectric to electrical conductivities,

$\mathcal{S}_{0}^{\mathrm{IM}}=\frac{\alpha_{0}^{\mathrm{IM}}}{\sigma_{0}^{\mathrm{IM}}}=\frac{\int_{-\infty}^{\infty} \frac{d E_{1}}{2 \pi} E_{1} \operatorname{sech}^{2}\left(\frac{E_{1}}{2}\right)\left[A^{c}\left(E_{1}\right)\right]^{2}}{\int_{-\infty}^{\infty} \frac{d E_{1}}{2 \pi} \operatorname{sech}^{2}\left(\frac{E_{1}}{2}\right)\left[A^{c}\left(E_{1}\right)\right]^{2}}=2 \pi \mathcal{E}_{c}$.

This relationship between the thermopower and the spectral asymmetry $\mathcal{E}_{c}$ was also found in a different model of coupled SYK dots realized in Ref. [28]. The ratios in Eqs. (4.17) and (4.18) hold even for a finite small $M / N$, as the effect of a finite small $M / N$ is simply a rescaling of the Green's function $G^{c}$.
Let us describe the fate of magnetotransport in the IM regime. On a lattice, we have $\Lambda \nu(0) \sim 1$. Then, $J_{\mathrm{IM}}=g^{2} / J$, and the conduction electron self-energy is approximately $\sqrt{J_{\mathrm{IM}} T}$. We have $J_{\mathrm{IM}} T \gg t^{2} \sim \Lambda^{2}$, so, to leading order, we can neglect the dispersion in Fermion propagators. Then, there is nothing for the magnetic field to couple to, and consequently no magnetotransport.

To illustrate this, let us compute the correlator of currents in perpendicular directions in real space on a square lattice. The uniform current operators are

$$
\begin{aligned}
I_{x}(\tau) \equiv & \frac{1}{V^{1 / 2}} \sum_{r} I_{r x}(\tau) \equiv-\frac{i t}{2 V^{1 / 2}} \sum_{r ; i=1}^{M} c_{r+\hat{x}, i}^{\dagger}(\tau) c_{r i}(\tau)+\text { H.c. } \\
I_{y}(\tau) \equiv & \frac{1}{V^{1 / 2}} \sum_{r} I_{r y}(\tau) \equiv-\frac{i t}{2 V^{1 / 2}} \sum_{r ; i=1}^{M} c_{r+\hat{y}, i}^{\dagger}(\tau) c_{r i}(\tau) e^{i \phi(r)} \\
& + \text { H.c. }
\end{aligned}
$$


where we have used a gauge with the magnetic vector potential $\mathbf{A}_{r}$ pointing along the $y$ direction, giving rise to the phase factors $e^{i \phi(r)}$ on bonds in the $y$ direction. The system volume in units of the unit cell volume is $V$. We then have

$$
\begin{aligned}
& \mathcal{T}_{\tau}\left\langle I_{x}(\tau) I_{y}\left(\tau^{\prime}\right)\right\rangle \\
&=-M \frac{t^{2}}{4 V} \sum_{r r^{\prime}}\left[\mathcal{T}_{\tau}\left\langle c_{r+\hat{x}}^{\dagger}(\tau) c_{r}(\tau) c_{r^{\prime}+\hat{y}}^{\dagger}\left(\tau^{\prime}\right) c_{r}^{\prime}\left(\tau^{\prime}\right) e^{i \phi\left(r^{\prime}\right)}\right\rangle\right. \\
&-\mathcal{T}_{\tau}\left\langle c_{r+\hat{x}}^{\dagger}(\tau) c_{r}(\tau) c_{r^{\prime}}^{\dagger}\left(\tau^{\prime}\right) c_{r^{\prime}+\hat{y}}\left(\tau^{\prime}\right) e^{-i \phi\left(r^{\prime}\right)}\right\rangle \\
&-\mathcal{T}_{\tau}\left\langle c_{r}^{\dagger}(\tau) c_{r+\hat{x}}(\tau) c_{r^{\prime}+\hat{y}}^{\dagger}\left(\tau^{\prime}\right) c_{r^{\prime}}\left(\tau^{\prime}\right) e^{i \phi\left(r^{\prime}\right)}\right\rangle \\
&\left.+\mathcal{T}_{\tau}\left\langle c_{r}^{\dagger}(\tau) c_{r+\hat{x}}(\tau) c_{r^{\prime}}^{\dagger}\left(\tau^{\prime}\right) c_{r^{\prime}+\hat{y}}\left(\tau^{\prime}\right) e^{-i \phi\left(r^{\prime}\right)}\right\rangle\right]
\end{aligned}
$$

where we have dropped the sum over flavor indices in favor of a global factor of $M$, and $\mathcal{T}$ denotes time ordering. To leading order in $t$, since the $c$ Green's functions are completely local,

$$
\mathcal{T}_{\tau}\left\langle c_{r}(\tau) c_{r^{\prime}}^{\dagger}\left(\tau^{\prime}\right)\right\rangle=\delta_{r r^{\prime}} G^{c}\left(\tau-\tau^{\prime}\right),
$$

none of the terms in Eq. (4.20) can be nonzero. Similarly, at $\mathcal{O}\left(t^{2}\right)$, there is no field-dependent correction to the $\left\langle I_{x} I_{x}\right\rangle$ correlator.

Perturbing in $t$, in order for Eq. (4.20) to be nonzero, we need to insert hopping vertices in order to close the fourpoint correlation functions of the $c$ 's. To lowest order in $t$, this requires insertion of two hopping vertices into each of the four-point correlation functions in Eq. (4.20), so that the connected contractions of $c$ 's and $c^{\dagger}$ 's into local $c$ Green's functions go around a single plaquette of the lattice. Again, because of our choice of gauge, hopping vertices along bonds in the $y$ direction come with phase factors. But we obtain, as we should, a gauge-invariant answer for the connected part, which is of interest to us here (the electrons are negatively charged, and $\mathcal{B}$ is defined in terms of $B$ as in Sec. IVA):

$$
\begin{aligned}
\left\langle I_{x} I_{y}\right\rangle\left(i \Omega_{m}\right)= & -i M \sin (\mathcal{B}) t^{4} T \sum_{\omega_{n}}\left(\left[G^{c}\left(i \omega_{n}\right)\right]^{3}\right. \\
& \left.\times\left[G^{c}\left(i \omega_{n}+i \Omega_{m}\right)-G^{c}\left(i \omega_{n}-i \Omega_{m}\right)\right]\right) .
\end{aligned}
$$

At $\mathcal{O}\left(t^{4}\right)$, vertex corrections from the coupling $g$ to this leading contribution vanish due to the noncorrelation of $g$ between distinct lattice sites, i.e., $\left\langle\left\langle g_{i j k l}^{r} g_{j i l k}^{r^{\prime}}\right\rangle\right\rangle=g^{2} \delta_{r r^{\prime}}$.

The DC Hall conductivity follows,

$$
\begin{aligned}
\sigma_{H}^{\mathrm{IM}}= & -\lim _{\omega \rightarrow 0} \frac{1}{i \omega}\left[\left\langle I_{x} I_{y}\right\rangle\left(i \Omega_{m} \rightarrow \omega+i 0^{+}\right)\right. \\
& \left.-\left\langle I_{x} I_{y}\right\rangle\left(i \Omega_{m} \rightarrow 0+i 0^{+}\right)\right] \\
= & 2 M \sin (\mathcal{B}) t^{4} \mathcal{P} \int_{-\infty}^{\infty} \frac{d E_{1}}{2 \pi} \frac{d E_{2}}{2 \pi} A_{3}^{c}\left(E_{1}\right) A^{c}\left(E_{2}\right) \\
& \times \frac{n_{f}\left(E_{2}\right)-n_{f}\left(E_{1}\right)}{\left(E_{2}-E_{1}\right)^{2}},
\end{aligned}
$$

where $\mathcal{P}$ denotes the Cauchy principal value, and

$$
\begin{aligned}
A_{3}^{c}\left(E_{1}\right) \equiv & -2 \operatorname{Im}\left(\left[G^{c}\left(i \omega_{n} \rightarrow E_{1}+i 0^{+}\right)\right]^{3}\right) \\
= & \operatorname{Im}\left[\frac{(i-1)\left(i+e^{2 \pi \mathcal{E}_{c}}\right)^{3} \cosh ^{3 / 4}(2 \pi \mathcal{E})}{2^{5 / 2} \pi^{9 / 4} J_{\mathrm{IM}}^{3 / 2} T^{3 / 2}\left(1+e^{4 \pi \mathcal{E}_{c}}\right)^{3 / 2}}\right. \\
& \left.\times \frac{\Gamma^{3}\left(\frac{1}{4}-\frac{i\left(E_{1}-2 \pi \mathcal{E}_{c} T\right)}{2 \pi T}\right)}{\Gamma^{3}\left(\frac{3}{4}-\frac{i\left(E_{1}-2 \pi \mathcal{E}_{c} T\right)}{2 \pi T}\right)}\right]
\end{aligned}
$$

is the spectral function of $\left[G^{c}\left(i \omega_{n}\right)\right]^{3}$. If $\mathcal{E}_{c}=0$, then the Hall conductivity vanishes due to the evenness of the spectral functions $A^{c}$ and $A_{3}^{c}$. This corresponds to half-filling the square lattice, so this is expected. Scaling out $T$ and evaluating the integral numerically gives

$$
\sigma_{H}^{\mathrm{IM}}=-M \sin (\mathcal{B}) \frac{t^{4} \cosh (2 \pi \mathcal{E})}{J_{\mathrm{IM}}^{2} T^{2}} \Xi_{H}^{\mathrm{IM}}\left(\mathcal{E}_{c}\right),
$$

where $\Xi_{H}^{\mathrm{IM}}\left(\mathcal{E}_{c}\right)$ is odd in $\mathcal{E}_{c}$, positive for positive $\mathcal{E}_{c}$, and vanishes when $\mathcal{E}_{c}=0, \pm \infty$. This is a very small contribution regardless of $B$; the already small flux per unit cell $\mathcal{B}$ is further multiplied by a small parameter $t^{4} /\left(J_{\mathrm{IM}}^{2} T^{2}\right)$. Note that we consider $\cosh (2 \pi \mathcal{E})$ to be $\mathcal{O}(1)$. If $|\mathcal{E}|$ is very large, then the conduction electrons do not scatter effectively off the SYK islands, as discussed before, and our perturbative expansion in hopping is no longer valid. In that case, the system is once again described by the MFL. For the Hall conductivity to be comparable to the longitudinal conductivity $\sigma_{0}^{\mathrm{IM}} \sim t^{2} /\left(J_{\mathrm{IM}} T\right)$, we need $\sin (\mathcal{B}) \sim J_{\mathrm{IM}} T / t^{2} \gg 1$, which is not even mathematically possible.

Similarly, the field-dependent correction to the $I_{x}-I_{x}$ correlator is

$$
\begin{aligned}
& \Delta_{B}\left[\left\langle I_{x} I_{x}\right\rangle\left(i \Omega_{m}\right)\right] \\
& =-M t^{4} \cos (\mathcal{B}) T \sum_{\omega_{n}}\left[G^{c}\left(i \omega_{n}\right)\right]^{2}\left[G^{c}\left(i \omega_{n}+i \Omega_{m}\right)\right]^{2},
\end{aligned}
$$

leading to the field-dependent correction to the longitudinal conductivity

$\Delta_{B}\left[\sigma_{L}^{\mathrm{IM}}\right]=\frac{M}{8} \frac{t^{4}}{T} \cos (\mathcal{B}) \int \frac{d E_{1}}{2 \pi} A_{2}^{c}\left(E_{1}\right) \operatorname{sech}^{2}\left(\frac{E_{1}}{2 T}\right)$,

where

$$
\begin{aligned}
& A_{2}^{c}\left(E_{1}\right) \\
& \equiv-2 \operatorname{Im}\left(\left[G^{c}\left(i \omega_{n} \rightarrow E_{1}+i 0^{+}\right)\right]^{2}\right) \\
& =-\operatorname{Im}\left[i \frac{\left(i+e^{2 \pi \mathcal{E}_{c}}\right)^{2} \cosh ^{1 / 2}(2 \pi \mathcal{E})}{2 \pi^{3 / 2} J_{\mathrm{IM}} T\left(1+e^{4 \pi \mathcal{E}_{c}}\right)} \frac{\Gamma^{2}\left(\frac{1}{4}-\frac{i\left(E_{1}-2 \pi \mathcal{E}_{c} T\right)}{2 \pi T}\right)}{\Gamma^{2}\left(\frac{3}{4}-\frac{i\left(E_{1}-2 \pi \mathcal{E}_{c} T\right)}{2 \pi T}\right)}\right],
\end{aligned}
$$

is the spectral function of $\left[G^{c}\left(i \omega_{n}\right)\right]^{2}$. Scaling out $T$ and evaluating the integral numerically gives 

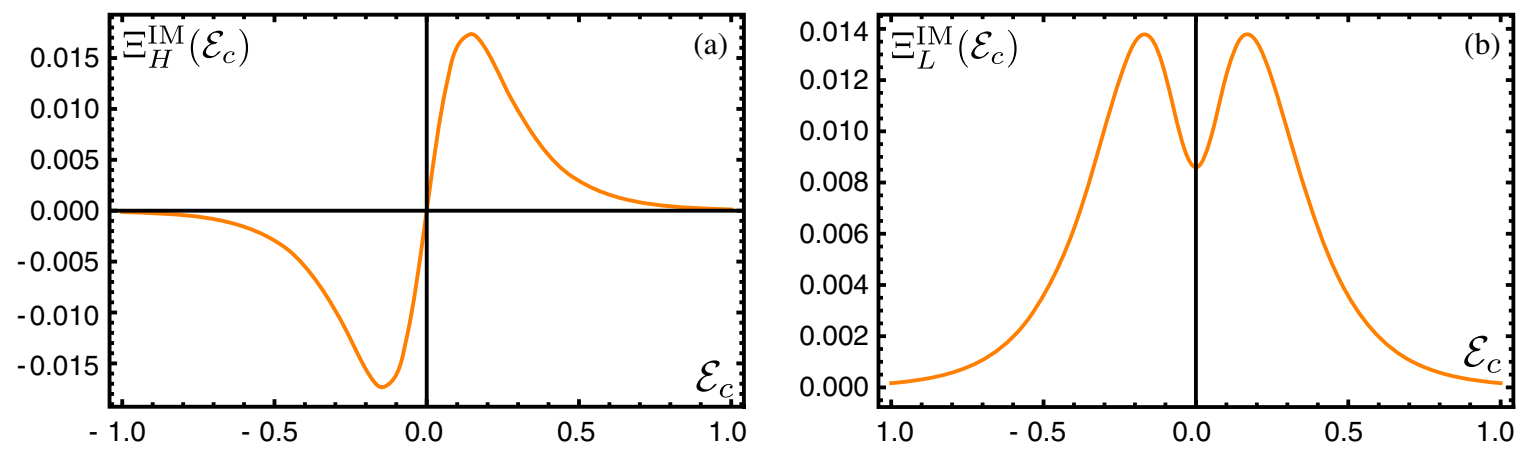

FIG. 3. Plots of (a) $\Xi_{H}^{\mathrm{IM}}\left(\mathcal{E}_{c}\right)$ and (b) $\Xi_{L}^{\mathrm{IM}}\left(\mathcal{E}_{c}\right)$. Both functions vanish in the limits of the fully filled and empty lattice $\left(\mathcal{E}_{c}=\mp \infty\right.$ respectively), as they should.

$$
\Delta_{B}\left[\sigma_{L}^{\mathrm{IM}}\right]=M \frac{t^{4} \cosh (2 \pi \mathcal{E})}{J_{\mathrm{IM}}^{2} T^{2}} \cos (\mathcal{B}) \Xi_{L}^{\mathrm{IM}}\left(\mathcal{E}_{c}\right)
$$

where $\Xi_{L}^{\mathrm{IM}}\left(\mathcal{E}_{c}\right)$ is even in $\mathcal{E}_{c}$, positive and nonzero for $\mathcal{E}_{c}=0$, and vanishes as $\mathcal{E}_{c} \rightarrow \pm \infty$. The longitudinal conductivity is, thus, reduced when a field is applied, as is usually the case.

It is similarly not possible to get a field-dependent correction to $\sigma_{L}^{\mathrm{IM}}$ that is comparable to its zero-field value. Thus, we shall no longer consider the IM regime for studying magnetotransport, as there is no qualitative difference between the regimes of large and small $B$, unlike in the MFL regime. For completeness, the plots of $\Xi_{H, L}^{\mathrm{IM}}\left(\mathcal{E}_{c}\right)$ are shown in Fig. 3.

Before we close this section, let us comment on the controllability of the hopping expansion used to compute the nonzero field-dependent conductivity corrections. Clearly, this hopping expansion must break down when $t$ is large enough, as the MFL has a very different conductivity tensor. Going from Eq. (4.20) to Eqs. (4.22) and (4.26), we only kept those $r^{\prime}$ relative to $r$ that resulted in $\mathcal{O}\left(t^{4}\right)$ corrections for the shortest closed paths from $r$ to $r^{\prime}$ and back. For arbitrary $r^{\prime}$, one can draw infinitely many paths that go from $r$ to $r^{\prime}$ and back. These paths may also intersect themselves in general. For a path length $l$, there are $<4^{l}$ paths for large $l$ as, at each step, one has four choices of direction, and not all possibilities will result in a formation of the closed path from $r$ to $r^{\prime}$ and back. Each step involves multiplying an additional local Green's function and factor of $t$, or roughly a factor of $t /\left(J_{\mathrm{IM}} T\right)^{1 / 2} \ll 1$ into the amplitude. Therefore, the total weight of paths of length $l$ should be $<\left(4 t /\left(J_{\mathrm{IM}} T\right)^{1 / 2}\right)^{l}$. The total weight of all paths between $r$ and $r^{\prime}$ then is $<\sum_{l=l_{\min }}^{\infty}\left[4 t /\left(J_{\mathrm{IM}} T\right)^{1 / 2}\right]^{l}=$ $\left[4 t /\left(J_{\mathrm{IM}} T\right)^{1 / 2}\right]^{l_{\min }} /\left[1-4 t /\left(J_{\mathrm{IM}} T\right)^{1 / 2}\right]$, where $l_{\min }$ is the length of the shortest closed path between $r$ and $r^{\prime}$, which scales as the lattice distance between $r$ and $r^{\prime}$. Thus, for $t /\left(J_{\mathrm{IM}} T\right)^{1 / 2} \ll 1$, the expansion is well behaved: As $r^{\prime}$ gets further away from $r$, the terms are exponentially suppressed in the distance between $r$ and $r^{\prime}$, whereas the number of $r^{\prime}$ s a given distance away from $r$ grows only linearly in that distance in two dimensions. Unsurprisingly, this is just the condition $T \gg T_{\mathrm{inc}}$ that we obtained earlier for the crossover into the IM regime.

\section{MACROSCOPIC TRANSPORT VIA EFFECTIVE- MEDIUM/RANDOM-RESISTOR THEORY}

We now return to the MFL with $B / T$ scaling that was described in Sec. IVA. We will show here that adding macroscopic disorder leads to a linear-in- $B$ magnetoresistance at large $B$, while preserving the $B / T$ scaling. We will treat the inhomogeneity in a classical transport framework. The quantum computation in Sec. IV A is used to compute a local $\sigma_{x x}$ and $\sigma_{x y}$, which is then input into a computation of global transport in a disordered sample by composing resistivities using Ohm's and Kirchhoff's laws.

\section{A. Setup}

We seek to understand the effects of additional macroscopic disorder on the transport of charge in the MFL at large magnetic fields $B$, in two spatial dimensions. This additional macroscopic disorder leads to the variation of the local conductivity tensor $\sigma(\mathbf{x})$ across the sample. Since the conduction electrons in our model interact with valence electrons in the SYK islands through a nontranslationally invariant interaction microscopically, the Navier-Stokes equation of hydrodynamics that describes dynamics of a nearly conserved macroscopic momentum [50] is not applicable to us, since this requires microscopic equilibration of the electron fluid through momentum-conserving interactions. (The effects of weak disorder on the magnetoresistance of a generic electron fluid with macroscopic momentum were studied in Ref. [51]. They did not find any regimes of linear magnetoresistance, instead finding that the magnetoresistance was quadratic with a prefactor controlled by the fluid viscosity.) Thus, at the coarse-grained level, we just have the equation for charge conservation, and Ohm's law:

$$
\nabla \cdot \mathbf{I}(\mathbf{x})=0, \quad \mathbf{I}(\mathbf{x})=\sigma(\mathbf{x}) \cdot \mathbf{E}(\mathbf{x}), \quad \mathbf{E}(\mathbf{x})=-\nabla \Phi(\mathbf{x}) .
$$


The effective local electric field $\mathbf{E}(\mathbf{x})$ (which includes the effects of Coulomb potentials generated due to charge inhomogeneities [52]) fluctuates spatially due to the macroscopic disorder, but equals an applied external electric field $\mathbf{E}_{0}=\langle\mathbf{E}(\mathbf{x})\rangle \equiv(1 / V) \int d^{2} \mathbf{x E}(\mathbf{x})$ on spatial average. We define the global conductivity tensor $\sigma^{e}$ through the relation $\langle\mathbf{I}(\mathbf{x})\rangle=\sigma^{e} \cdot \mathbf{E}_{0}$ and parametrize the deviation $\sigma(\mathbf{x})-\sigma^{e}=\delta \sigma(\mathbf{x})$. The condition $\langle\mathbf{I}(\mathbf{x})-\langle\mathbf{I}(\mathbf{x})\rangle\rangle=0$ then gives $\left\langle\chi(\mathbf{x}) \cdot \mathbf{E}_{0} \equiv \delta \sigma(\mathbf{x}) \cdot \mathbf{E}(\mathbf{x})\right\rangle=0$.

Following Ref. [35], without making any additional approximations, the solution of these equations can be formally cast in the form

$\Phi(\mathbf{x})=-\mathbf{E}_{0} \cdot \mathbf{x}+\int d^{2} \mathbf{x}^{\prime} \mathcal{G}\left(\mathbf{x}, \mathbf{x}^{\prime}\right) \nabla^{\prime} \cdot\left[\delta \sigma\left(\mathbf{x}^{\prime}\right) \cdot \nabla^{\prime} \Phi\left(\mathbf{x}^{\prime}\right)\right]$,

where the Green's function satisfies $\nabla \cdot\left[\sigma^{e} \cdot \nabla \mathcal{G}\left(\mathbf{x}, \mathbf{x}^{\prime}\right)\right]=$ $-\delta\left(\mathbf{x}-\mathbf{x}^{\prime}\right), \mathcal{G}\left(\mathbf{x}, \mathbf{x}^{\prime}\right)=\mathcal{G}\left(\mathbf{x}^{\prime}, \mathbf{x}\right)$, and $G\left(\mathbf{x}, \mathbf{x}^{\prime} \in \partial V\right)=0$, for the system boundary $\partial V$, which we take to infinity. Taking a gradient on both sides, we get

$$
\begin{aligned}
& \mathbf{E}(\mathbf{x})=\mathbf{E}_{0}-\int d^{2} \mathbf{x}^{\prime}\left(\left[\delta \sigma\left(\mathbf{x}^{\prime}\right) \cdot \mathbf{E}\left(\mathbf{x}^{\prime}\right)\right] \cdot \nabla^{\prime}\right) \cdot \nabla \mathcal{G}\left(\mathbf{x}, \mathbf{x}^{\prime}\right), \text { or } \\
& \chi(\mathbf{x})=\delta \sigma(\mathbf{x})-\delta \sigma(\mathbf{x}) \cdot \int d^{2} \mathbf{x}^{\prime} \mathcal{K}\left(\mathbf{x}, \mathbf{x}^{\prime}\right) \cdot \chi\left(\mathbf{x}^{\prime}\right),
\end{aligned}
$$

where the second line follows from the first by leftmultiplying both sides by $\delta \sigma(\mathbf{x})$, and then demanding that it hold for any $\mathbf{E}_{0}$, and $\mathcal{K}_{i j}\left(\mathbf{x}, \mathbf{x}^{\prime}\right)=\partial_{i} \partial_{j}^{\prime} \mathcal{G}\left(\mathbf{x}, \mathbf{x}^{\prime}\right)$.

We now assume that the disorder divides the sample into macroscopic domains whose size is much smaller than the sample size, but much bigger than the smaller of the electron mean-free path and electron cyclotron radius, and the tensors $\chi$ and $\delta \sigma$ take on constant values in a given domain. For a given domain $p$, we can write

$$
\begin{aligned}
\chi^{p}= & \delta \sigma^{p}-\delta \sigma^{p} \cdot \int_{p} d^{2} \mathbf{x}^{\prime} \mathcal{K}\left(\mathbf{x} \in p, \mathbf{x}^{\prime}\right) \cdot \chi^{p} \\
& -\delta \sigma^{p} \cdot \sum_{p^{\prime} \neq p} \cdot \int_{p^{\prime}} d^{2} \mathbf{x}^{\prime} \mathcal{K}\left(\mathbf{x} \in p, \mathbf{x}^{\prime}\right) \cdot \boldsymbol{\chi}^{p^{\prime}}
\end{aligned}
$$

For the second integral over domains other than the given domain, we replace $\boldsymbol{\chi}^{n}$ with its spatial average $\langle\boldsymbol{\chi}\rangle$. This is the "effective-medium" approximation [35]: The equivalent conductivity of each domain is controlled in part by a "mean field" of domains surrounding it. However, since our conventions are set up so that $\langle\boldsymbol{x}\rangle=0$, this second term drops out. Then, spatially averaging both sides, we obtain

$$
\sum_{p} V^{p} \boldsymbol{\chi}^{p}=0 \Rightarrow \sum_{p} V^{p}\left(\mathbb{I}+\delta \sigma^{p} \cdot \mathcal{M}^{p}\right)^{-1} \cdot \delta \sigma^{p}=0,
$$

where $V^{p}$ is the volume fraction of domain $p$ and $\mathcal{M}_{i j}^{p}=$ $\oint_{\partial^{\prime} p} \partial_{i} \mathcal{G}\left(\mathbf{x}, \mathbf{x}^{\prime}\right) \hat{n}_{j}^{\prime p}$, where the integral is over the primed coordinate, and $\hat{\mathbf{n}}^{\prime p}$ is the outward-pointing unit normal vector on the boundary of $p$, varying with the primed coordinate.

If the local conductivity tensor $\sigma(\mathbf{x})$ is known in all domains, Eq. (5.5) can then be solved for $\sigma^{e}$. In our twodimensional electron problem, we expect $\sigma_{i j}^{e}=\delta_{i j} \sigma_{L}^{e}-\epsilon_{i j} \sigma_{H}^{e}$, where $\sigma_{L}^{e}$ is even in $B$ and $\sigma_{H}^{e}$ is odd in $B$ because of Onsager reciprocity, so we obtain the Green's function $\mathcal{G}\left(\mathbf{x}, \mathbf{x}^{\prime}\right)=-\ln \left(\left|\mathbf{x}-\mathbf{x}^{\prime}\right| 0^{+}\right) /\left(2 \pi \sigma_{L}^{e}\right)$. Then, for circular domains, $\mathcal{M}_{i j}^{p}=\delta_{i j} /\left(2 \sigma_{L}^{e}\right)$ is indeed independent of $\mathbf{x}$. This makes Eqs. (5.4) and (5.5) self-consistent [35]. For other domain shapes, there are corrections when $\mathbf{x}$ is near the domain boundary.

For an analytically solvable toy model, we assume that the $\sigma(\mathbf{x})$ can take either of two possible values $\sigma^{a}$ and $\sigma^{b}$ in circular domains that are spatially randomly distributed over the sample [34,38] [Fig. 4(a)]. As far as the asymptotic low- and high-field magnetoresistance goes, this already yields the same qualitative behavior at large and small fields as a more complicated model with a distribution of different types of domains [40]. Furthermore, the meanfield-like effective-medium approximation has also been shown to produce results for the magnetoresistance equivalent to exact numerical solutions of Eq. (5.1) in randomresistor network models $[36,37,40]$. In the simplified two-type scenario, Eq. (5.5) then reduces to [38]

$$
\begin{aligned}
& V^{a}\left(\mathbb{I}+\frac{\sigma^{a}-\sigma^{e}}{2 \sigma_{L}^{e}}\right)^{-1} \cdot\left(\sigma^{a}-\sigma^{e}\right) \\
& \quad+\left(1-V^{a}\right)\left(\mathbb{I}+\frac{\sigma^{b}-\sigma^{e}}{2 \sigma_{L}^{e}}\right)^{-1} \cdot\left(\sigma^{b}-\sigma^{e}\right)=0 .
\end{aligned}
$$

If $V^{a}=1 / 2$, this yields an unsaturating high-field linear magnetoresistance [38]. For the model with a distribution of domains, the equivalent condition is that the distribution is symmetric about its mean [40]. For $V^{a}$ detuned from $1 / 2$, the magnetoresistance saturates, but there is an intermediate regime of fields in which the magnetoresistance is approximately linear, and the saturation field becomes arbitrarily large as $V^{a}$ approaches $1 / 2$ [38]. The rough reasoning behind the saturation appears to be that, if one type of domain is far more common than the other, the current flowing through the sample mainly finds paths involving only one type of domain. Hence, the global magnetoresistance behaves like that of a single domain, which saturates at high fields [37]. We will do our analysis with the symmetric distribution $V^{a}=1-V^{a}=1 / 2$.

A physical picture for the high-field linear magnetoresistance was provided in Ref. [36] and involves the contribution of the local Hall resistance (which is linear in $B$ ) to the global longitudinal resistance due to the distortion in current paths arising from spatial fluctuations of the local Hall resistance: In a uniform sample, charge accumulation at the edges of the sample parallel to the applied electric field produces a global Hall electric field perpendicular to the applied electric field that cancels out Hall currents 


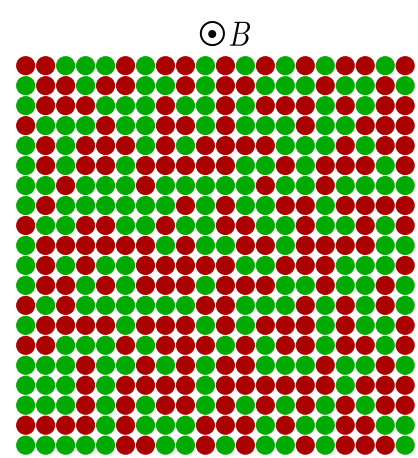

(a)

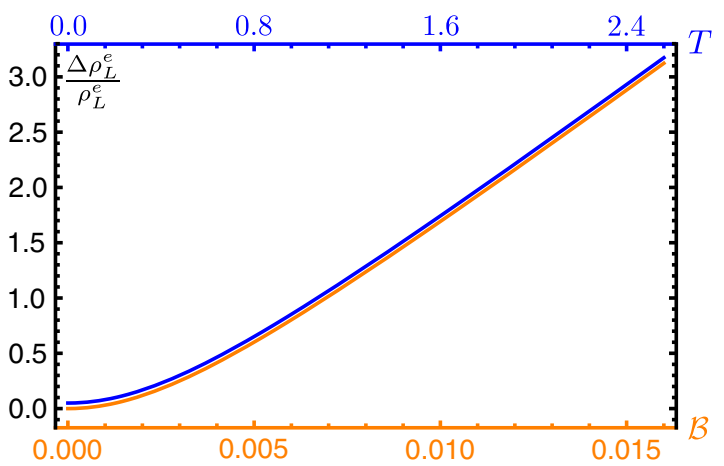

(b)

FIG. 4. (a) A two-dimensional sample with a random distribution of approximately equal fractions of two types of domains, for which an exact analytic solution of the effective-medium equations for magnetotransport is possible. The magnetic field $B$ points out of the plane of the sample. (b) Plots of the normalized change in global longitudinal resistance due to dimensionless magnetic field $\mathcal{B}$ (orange) and due to temperature $T$ (blue), obtained from Eq. (5.9). We use $E_{b}^{F} / E_{a}^{F}=0.8$ and $\gamma_{b} / \gamma_{a}=0.8$. The dimensionless magnetic field $\mathcal{B}$ is the flux per unit cell $B a^{2}$ in units of $\hbar / e$ [Eq. (4.8)]. We use $m=0.005 \sim 1 / E_{a, b}^{F}$. The orange $(\mathcal{B})$ curve is evaluated at $T=1.0$ and $\gamma_{a}=0.1$, and the blue $(T)$ curve is evaluated at $\mathcal{B}=0.0025$ and $\gamma_{a}=0.1 T$. The curves are slightly offset for visualization, but actually lie on top of each other, demonstrating a scaling between magnetic field and temperature. Both the $\mathcal{B}$ and $T$ dependencies are quadratic at small fields or temperatures and cross over to linear at large fields or temperatures.

throughout the sample. On the other hand, if the sample has a disordered local conductivity tensor, the global Hall electric field no longer cancels out local Hall currents throughout the sample. Thus, the global longitudinal resistance becomes dependent on the local Hall resistances.

\section{B. Application}

We note that, in Eq. (4.11), the sech is strongly peaked near $E_{1}=0$, whereas for a finite temperature, $\operatorname{Im}\left[\Sigma_{R}^{c}\left(E_{1}\right)\right]$ does not vary drastically with $E_{1}$ near $E_{1}=0$ over the range in which the sech is appreciable. We can, thus, replace $\operatorname{Im}\left[\Sigma_{R}^{c}\left(E_{1}\right)\right]$ with $\gamma / 2$ from Eq. (3.9). Regardless of this approximation, we note from Eq. (4.11) that $\sigma_{L}^{\mathrm{MFL}} \sim$ $T / B^{2}$ and $\sigma_{H}^{\mathrm{MFL}} \sim 1 / B$ at large $B$, which is what the effective-medium theory needs to produce linear magnetoresistance at large $B$. This asymptotic scaling holds even if we had multiple MFL bands, thus adding their conductivity tensors to get the appropriate local conductivity tensor.

We thus input the following conductivity tensors into the effective-medium calculation (we take the band mass $m=$ $k_{F} / v_{F}$ to be the same in both types of electronlike domains $a$ and $b$ ):

$$
\sigma_{i j}^{a, b}=\frac{\sigma_{0 a, b}^{\mathrm{MFL}}}{1+\mathcal{B}^{2} /\left(m \gamma_{a, b}\right)^{2}}\left(\delta_{i j}+\epsilon_{i j} \frac{\mathcal{B}}{m \gamma_{a, b}}\right) .
$$

The scattering rate $\gamma$ can fluctuate across domains due to fluctuations in $g$, induced by fluctuations in the densities of SYK islands, and the base conductivity $\sigma_{0 a, b}^{\mathrm{MFL}}$ can fluctuate across domains due to fluctuations in both $g$ and in the electron density. Then, solving Eq. (5.6) for $V^{a}=1-V^{a}=$ $1 / 2$, we get the global longitudinal and Hall resistances, respectively,

$$
\begin{aligned}
& \rho_{L}^{e} \equiv \frac{\sigma_{L}^{e}}{\sigma_{L}^{e 2}+\sigma_{H}^{e 2}}=\frac{\sqrt{(\mathcal{B} / m)^{2}\left(\gamma_{a} \sigma_{0 a}^{\mathrm{MFL}}-\gamma_{b} \sigma_{0 b}^{\mathrm{MFL}}\right)^{2}+\gamma_{a}^{2} \gamma_{b}^{2}\left(\sigma_{0 a}^{\mathrm{MFL}}+\sigma_{0 b}^{\mathrm{MFL}}\right)^{2}}}{\gamma_{a} \gamma_{b}\left(\sigma_{0 a}^{\mathrm{MFL}} \sigma_{0 b}^{\mathrm{MFL}}\right)^{1 / 2}\left(\sigma_{0 a}^{\mathrm{MFL}}+\sigma_{0 b}^{\mathrm{MFL}}\right)}, \\
& \rho_{H}^{e} \equiv-\frac{\sigma_{H}^{e} / \mathcal{B}}{\sigma_{L}^{e 2}+\sigma_{H}^{e 2}}=\frac{\gamma_{a}+\gamma_{b}}{m \gamma_{a} \gamma_{b}\left(\sigma_{0 a}^{\mathrm{MFL}}+\sigma_{0 b}^{\mathrm{MFL}}\right)} .
\end{aligned}
$$

The magnetoresistance $\rho_{L}^{e}(\mathcal{B})-\rho_{L}^{e}(0)$ is thus linear as promised at high fields and is quadratic at low fields.

Considering the isotropic parabolic dispersion $\varepsilon_{k}=k^{2} /(2 m)-\Lambda / 2$, and using Eq. (4.3), Eq. (3.9), and $\nu(0)=m$, we can write $\sigma_{0 a, b}^{\mathrm{MFL}}=M w_{\sigma} E_{F}^{a, b} / \gamma_{a, b}$, where $w_{\sigma}=0.135689$ and $E_{F}^{a, b}=m v_{F a, b}^{2} / 2$ are the Fermi energies. We can then rewrite Eq. (5.8) as

$$
w_{\sigma} \rho_{L}^{e}=\frac{\left(\gamma_{a}^{2}+\left(\frac{\mathcal{B}}{m}\right)^{2} \frac{\left(1-E_{b}^{F} / E_{a}^{F}\right)^{2}}{\left(\gamma_{b} / \gamma_{a}+E_{b}^{F} / E_{a}^{F}\right)^{2}}\right)^{1 / 2}}{M\left(\gamma_{a} / \gamma_{b}\right)^{1 / 2}\left(E_{a}^{F} E_{b}^{F}\right)^{1 / 2}}, \quad w_{\sigma} \rho_{H}^{e}=\frac{\left(1+\gamma_{b} / \gamma_{a}\right)}{M m E_{a}^{F}\left(E_{b}^{F} / E_{a}^{F}+\gamma_{b} / \gamma_{a}\right)}
$$


Plots of the normalized change in $\rho_{L}^{e}$ due to $\mathcal{B}$ and $T$ are shown in Fig. 4(b). This simplified model with two types of domains thus leads to a global longitudinal resistance that adds $T$ and $B$ in quadrature, [53] as seen in the experiment of Ref. [5]. A continuous Gaussian distribution of electron densities across the domains will also yield a qualitatively similar scaling function to the above quadrature function [40]. In general, the zero-field linear-in- $T$ and highfield linear-in- $B$ behavior (as well as the scaling between $B$ and $T$ ) will emerge universally from such resistor-network models, but the interpolation between the two regimes is sensitive to the distribution of the local conductivity tensors.

The Hall resistance $\rho_{H}^{e}$ is sensitive to the disorder distribution and, thus, is not trivially controlled by the average carrier density $\propto \operatorname{Mm}\left(E_{b}^{F}+E_{a}^{F}\right) / 2$, even for the isotropic Fermi surfaces we consider, unless $\gamma_{a}=\gamma_{b}$. In this simplified version of the problem, $\rho_{H}^{e}$ is independent of temperature. However, we expect that more complicated disorder distributions generically give rise to some temperature dependence of $\rho_{H}^{e}$, which would depend on the disorder distribution, even at a qualitative level. A detailed analysis of such effects is beyond the scope of the present work and will be considered in the future.

Since $\gamma_{a, b} \propto T$, the crossover from quadratic to linear magnetoresistance occurs at a field scale proportional to temperature. Additionally, if we use the Planckian choice of parameters, and if the disorder distribution is such that $\left|1-E_{b}^{F} / E_{a}^{F}\right| /\left(\gamma_{b} / \gamma_{a}+E_{b}^{F} / E_{a}^{F}\right)$ is an $\mathcal{O}(1)$ number, the crossover occurs at a field scale given by $\mu_{B} B \sim k_{B} T$, as discussed at the end of Sec. IVA. While this is most definitely a fine-tuned situation, and would require substantial variation in the charge densities between domains, it is within the scope of our theory. Alternatively, if $\gamma_{a}\left(\gamma_{b} / \gamma_{a}+E_{b}^{F} / E_{a}^{F}\right) /\left(k_{B} T\left|1-E_{b}^{F} / E_{a}^{F}\right|\right)$ is an $\mathcal{O}(1)$ quantity (but $\gamma_{a} \propto T$ is much smaller than $k_{B} T$ ), then $\rho_{L}^{e}$ can still be controlled by the approximate scaling function $\sqrt{1+\left(\mu_{B} B\right)^{2} /\left(k_{B} T\right)^{2}}$ for much smaller variations in the charge densities between domains.

The effective-medium theory is applicable when the domain sizes are much greater than the smaller of the electron mean-free path and electron cyclotron radius in a single domain. At low temperatures and weak fields, electrons can move through a domain without significant loss or deflection of momentum, and the effects of scattering off the boundaries between domains then become important, adding a temperature-independent residual resistivity to the result of the above computation.

In our analysis, we have neglected the effects of the feedback of heat currents on charge transport. In general, one would have an additional analogous set of equations to Eq. (5.1) for heat currents and temperature gradients in place of charge currents and electric fields. Since there is no concept of bulk fluid motion due to translational symmetry breaking at the microscopic level, the equations for heat currents and charge currents would only be coupled if the local thermoelectric tensor $\alpha(\mathbf{x})$ were nonzero. However, in the MFL, with $T \ll E_{a, b}^{F}, \alpha(\mathbf{x})$ is negligible, as discussed in Sec. IV A, and our decoupled analysis of charge currents is hence still applicable. Somewhere in the crossover region between the MFL and the IM, a regime may exist where both $\alpha(\mathbf{x})$ and the effects of magnetic fields on the local conductivity tensors are simultaneously significant, and there may be a significant feedback of thermoelectric effects on the charge magnetotransport. We leave a detailed study of such effects for future work.

\section{DISCUSSION}

The strange metal phases of the cuprate and pnictide high- $T_{c}$ superconductors occur at finite dopings and consequently display significant amounts of disorder. Experimentally, there is direct evidence for disorder at (i) microscopic levels, due to irregular placements of dopant atoms [55], and (ii) meso- and macroscopic levels, due to a variety of factors, ranging from crystalline imperfections to charge puddles caused by impurities and nonisovalent dopants [56,57]. Additionally, due to these materials being layered, with relatively poor interlayer conductivities, imperfections in a layer may further induce heterogeneities in the charge distributions of adjacent layers through Coulomb forces.

We have attempted to paint an impressionist picture of transport and magnetotransport in a strange metal by developing a solvable model that incorporates disorder at both the microscopic and macroscopic levels. At the microscopic level, we built off remarkable recent developments [10,27,28,33,58,59] in realizing solvable fieldtheoretic descriptions of extended non-Fermi liquid phases using SYK models. These models couple together SYK quantum dots without quasiparticle excitations and show how this can lead to non-Fermi liquid transport in an extended finite-dimensional phase. In our model, we locally and randomly couple mobile conduction electrons to immobile quantum dots described by SYK models in a particular way. In this manner, we realized a disordered MFL phase at low temperatures, with a linear-in- $T$ resistivity and an identifiable Fermi surface. We determined the two-point functions, conductivities, and magnetotransport properties of this phase exactly in two spatial dimensions, finding a scaling between magnetic field and temperature in the conductivity tensor. Additionally, we showed that nearly local IM phases, with no identifiable Fermi surface, are also realized in our model at higher temperatures in certain parameter regimes; these IMs can also have linearin- $T$ resistivities, but they have very weak effects of magnetic fields on their charge transport properties, making them unlikely candidates for a description of the strange metals seen in experiments at lower temperatures, which is where the large linear-in- $B$ magnetoresistances are also observed. However, the IMs may still be the correct concept at high temperatures, due to strong bad-metallic behavior 
displayed through their large resistivities, as is seen in experiments. It should also be noted that the large linear magnetoresistances are not observed in experiments performed at high temperatures where the system is a bad metal, with a zero-field resistivity much larger than the quantum unit $h / e^{2}[5,6]$, which is consistent with the behavior of an IM.

While the MFL regime of our model does indeed have a linear-in- $T$ resistivity, and also a $B / T$ scaling at approximately the observed $B$ scale, it yields a magnetoresistance that saturates at large $B$. To obtain a nonsaturating magnetoresistance, we argued for the importance of macroscopic disorder in the MFL regime. To model such effects, we applied the effective-medium approximation to a sample containing domains of our disordered linear-in- $T$ MFLs with varying electron densities. While the effectivemedium approximation is a mean-field theory at the level of Kirchhoff's and Ohm's laws for current flow, it has shown to be equivalent to exact numerical simulations of randomresistor networks for magnetotransport [40], and it has also had remarkable successes in describing experimentally observed magnetoresistances in other two-dimensional disordered materials [40,60,61]. For certain simplified disorder distributions, the effective-medium equations for magnetotransport are analytically solvable. These exactly solvable equations yield, in our case, a magnetoresistance that is quadratic in field at low fields, crosses over to linear in field at high fields, and is controlled by a scaling function between field and temperature, as seen in recent experiments on the pnictide and cuprate strange metals $[5,6]$.

On the experimental front, the anomalous high-field linear magnetoresistance in the cuprate and pnictide strange metals is already known to be dependent on the component of the magnetic field perpendicular to the sample plane [62], a feature that our model reproduces, since it is based on orbital effects of the magnetic field on charge transport. Furthermore, a strong linear component of the high-field magnetoresistance is seen even away from the critical doping at which the zero-field resistance is almost exactly linear in $T[5,6]$. The disorder based mechanism considered by us would be consistent with this observation, as the zerofield linear-in- $T$ behavior is not a prerequisite for high-field disorder-induced linear magnetoresistance; all that is required is that the local conductivity tensor behave like Eq. (5.7) as a function of the magnetic field.

On the theoretical front, we have been able to analytically calculate nontrivial magnetotransport properties in a somewhat contrived, but solvable, model of a disordered non-Fermi liquid. Studies along the lines of Refs. [29-31] could show how such models emerge naturally as effective theories of realistic, disordered, single-band Hubbard models. We hope that our study motivates further investigations into the interplay of disorder and strong interactions in the transport properties of the strange metal phases of the pnictides and cuprates.

\section{ACKNOWLEDGMENTS}

We thank J. G. Analytis, L. Balents, J. C. Séamus Davis, E.-A. Kim, A. Lucas, and B. Ramshaw for useful discussions. A. A. P. was supported by the NSF Grant No. PHY1125915 via a KITP Graduate Fellowship. This research was also supported by the NSF Grant No. DMR-1664842, by the MURI Grant No. W911NF-14-1-0003 from ARO, and by funds provided by the U.S. Department of Energy (DOE) under cooperative research agreement DESC0009919. Research at the Perimeter Institute is supported by the Government of Canada through Industry Canada and by the Province of Ontario through the Ministry of Research and Innovation. S. S. also acknowledges support from Cenovus Energy at the Perimeter Institute and from the Hanna Visiting Professor program at Stanford University.

Note added.-Recently, we learned of related but independent work by Chowdhury et al. on realizing translationally invariant microscopic models of non-Fermi liquids using SYK models [63].

\section{APPENDIX A: EFFECTS OF PAIR HOPPING AND BILINEAR TERMS ON THE MARGINAL-FERMI LIQUID}

We consider the effects of the pair-hopping term [Eq. (3.12)] on the MFL as $T \rightarrow 0$. With the Hamiltonian given by Eq. (3.12), the Dyson equations are given by

$$
\begin{aligned}
\Sigma(\tau)= & -J^{2} G^{2}(\tau) G(-\tau)-\frac{M}{N} g^{2} G(\tau) G^{c}(\tau) G^{c}(-\tau) \\
& -\frac{M}{N} \eta^{2} G(-\tau)\left[G^{c}(\tau)\right]^{2}, \\
G\left(i \omega_{n}\right)= & \frac{1}{i \omega_{n}+\mu-\Sigma\left(i \omega_{n}\right)}, \\
\Sigma^{c}(\tau)= & -g^{2} G^{c}(\tau) G(\tau) G(-\tau)-\eta^{2} G^{c}(-\tau)[G(\tau)]^{2}, \\
G^{c}\left(i \omega_{n}\right)= & \int \frac{d^{d} k}{(2 \pi)^{d}} \frac{1}{i \omega_{n}-\epsilon_{k}+\mu-\Sigma^{c}\left(i \omega_{n}\right)} .
\end{aligned}
$$

If $\mu=0$, the exact relations $G(\tau)=-G(-\tau)$ and $G^{c}(\tau)=$ $-G^{c}(-\tau)$ imply that the only effect of the pair-hopping term on the physics considered in the main text in all regimes is just a redefinition of $g$, with $g \rightarrow\left(g^{2}+\eta^{2}\right)^{1 / 2}$.

As long as the bandwidth is large, i.e., $t \gg g, \eta, J$, Eq. (3.3) is still valid. Following the same procedure as we did in Sec. III A, and using $G(\tau)$ given by Eq. (3.5), we obtain

$$
\begin{aligned}
\Sigma^{c}\left(i \omega_{n} \rightarrow 0\right)= & \frac{i g^{2} \nu(0)}{2 J \cosh ^{1 / 2}(2 \pi \mathcal{E}) \pi^{3 / 2}} \omega_{n} \ln \left(\frac{\left|\omega_{n}\right| e^{\gamma_{E}-1}}{J}\right) \\
& +\frac{\eta^{2} \nu(0) \cosh ^{1 / 2}(2 \pi \mathcal{E})}{2 \pi^{3 / 2}}\left(i \frac{\omega_{n}}{J} \ln \left(\frac{\left|\omega_{n}\right| e^{\gamma_{E}-1}}{J}\right)\right. \\
& -\tanh (2 \pi \mathcal{E}))+\mathcal{O}\left(\omega_{n}\right) .
\end{aligned}
$$


This is clearly a marginal-Fermi liquid with an additional chemical potential correction

$$
\delta \mu=\frac{\eta^{2} \nu(0) \cosh ^{1 / 2}(2 \pi \mathcal{E})}{2 \pi^{3 / 2}} \tanh (2 \pi \mathcal{E}) \ll \Lambda,
$$

which leads to a harmless small change in the size of the conduction electron Fermi surface, as the numbers of $c$ and $f$ electrons are no longer independently conserved (but their sum is conserved).

There is also a backreaction to the SYK dots,

$$
\begin{aligned}
& \tilde{\Sigma}(\tau)=-\frac{M}{N} g^{2} G(\tau) G^{c}(\tau) G^{c}(-\tau)-\frac{M}{N} \eta^{2} G(-\tau)\left[G^{c}(\tau)\right]^{2}, \\
& \tilde{\Sigma}\left(i \omega_{n} \rightarrow 0\right)=\frac{M g^{2}[\nu(0)]^{2} J \sinh (\pi \mathcal{E})}{3 \sqrt{2} N \pi^{9 / 4} \cosh ^{1 / 4}(2 \pi \mathcal{E})}+\mathcal{O}\left(\omega_{n}\right),
\end{aligned}
$$

which is again a chemical potential correction plus irrelevant frequency-dependent corrections. This chemical potential correction actually changes $\mathcal{E}$, which is no longer a conserved quantity, and it is determined by the condition $\operatorname{Re}\left[\Sigma\left(i \omega_{n} \rightarrow 0\right)\right]=\mu+\delta \mu$.

We also briefly discuss qualitatively the effects of certain fermion bilinears in (2.1). Terms bilinear in the $f$ 's destroy their SYK behavior and nonzero entropy as $T \rightarrow 0$. The $c$ 's then scatter off essentially noninteracting randommatrix islands, with $G\left(i \omega_{n}\right) \sim i \operatorname{sgn}\left(\omega_{n}\right)$. This leads to $\operatorname{Im}\left[\Sigma_{R}^{c}(0)\right] \sim T^{2}$, and the $c$ 's hence realize a weakly interacting disordered Fermi liquid as $T \rightarrow 0$. However, if the coefficients of the $f$-bilinears are small, then their SYK behavior is restored for temperatures larger than a small energy scale $E_{c}$ [10]. Hence, the marginal-Fermi liquid behavior of the $c$ 's is also restored for $T>E_{c}$.

The effects of bilinears which hybridize $c$ 's and $f$ 's (such as $c^{\dagger} f$ ) were discussed in Ref. [33]. In the $N \rightarrow \infty$ limit, these lead to $\operatorname{Im}\left[\Sigma_{R}^{c}(0)\right] \sim 1 / \sqrt{T}$ when the $f$ 's are described by SYK models. This is more relevant than the MFL selfenergy $(\sim T)$ at low $T$, but less relevant at high $T$. Thus, once again, if the coefficients of these bilinears are small, then the MFL self-energy will dominate above a certain temperature scale, and the MFL behavior will be restored.

\section{APPENDIX B: BOLTZMANN EQUATION FOR THE MARGINAL-FERMI LIQUID}

We provide a derivation of Eq. (4.6). We follow the notation, style, and mechanics of Chapter 5 of Ref. [46]. The general off-shell Boltzmann equation for modes close to the isotropic Fermi surface $\left(|p| \approx p_{F}\right.$; we do not use boldface for momentum-space vectors) is given by

$$
\begin{gathered}
-\left[\left(i \partial_{t}+v_{F}\left|\nabla+\mathbf{A}_{E}+\mathbf{A}_{B}\right|\right) \circ, F\right] \\
=\Sigma_{K}^{c}-\left(\Sigma_{R}^{c} \circ F-F \circ \Sigma_{A}^{c}\right),
\end{gathered}
$$

where $F(t, \mathbf{r}, p, \omega)=1-2\left[n_{f}(\omega)+\delta n(t, \mathbf{r}, p, \omega)\right]$ is a parametrization of the distribution function, and $\mathbf{A}_{E}(t)$ and $\mathbf{A}_{B}(\mathbf{r})$ are parts of the electromagnetic vector potential giving rise to the uniform electric and magnetic fields, respectively, with $-d \mathbf{A}_{E}(t) / d t=\mathbf{E}(t)$ and $\nabla \times \mathbf{A}_{B}(\mathbf{r})=\mathcal{B} \hat{z}$ ( $\nabla$ denotes the spatial gradient). $\Sigma_{R, A, K}^{c}$ are the retarded, advanced, and Keldysh components of the conduction electron self-energy, respectively. Equation (B1) follows from the Dyson equation for two-point functions on the Keldysh contour [46] and, hence, it is exact due to the large $M, N$ limits. The $\circ$ denotes the convolution,

$$
\begin{aligned}
Z & =X \circ Y \Rightarrow Z\left(t_{1}, \mathbf{r}_{1}, t_{2}, \mathbf{r}_{2}\right) \\
& =\int d t_{3} d^{2} \mathbf{r}_{3} X\left(t_{1}, \mathbf{r}_{1}, t_{3}, \mathbf{r}_{3}\right) Y\left(t_{3}, \mathbf{r}_{3}, t_{2}, \mathbf{r}_{2}\right),
\end{aligned}
$$

in the two-coordinate representation, and the $[.,$.$] denotes a$ commutator. We will, however, mostly use the centralrelative coordinate representation instead, with $p, \omega$ being Fourier transforms of the relative coordinate $\mathbf{r}_{1}-\mathbf{r}_{2}, t_{1}-t_{2}$, and $\mathbf{r}, t$ denoting the central coordinate $\left(\mathbf{r}_{1}+\mathbf{r}_{2}\right) / 2$, $\left(t_{1}+t_{2}\right) / 2$; this convolution can then be appropriately reexpressed in this representation following Ref. [46].

We then use a coordinate remapping $k=p+\mathbf{A}_{B}(\mathbf{r})[64,65]$ to redefine $F(t, \mathbf{r}, p, \omega)=1-2\left[n_{f}(\omega)+\delta n(t, \mathbf{r}, p, \omega)\right] \Rightarrow$ $F(t, k, \omega)=1-2\left[n_{f}(\omega)+\delta n(t, k, \omega)\right]$. This is valid as long as the Fermi energy is large enough to make the effects of Landau quantization insignificant at the fields in question. The only $\mathbf{r}$ dependence in $F$ then is fictitious, coming from the $\mathbf{r}$ dependence of $\mathbf{A}_{B}$, and should not affect physical results for spatially uniform transport quantities due to gauge invariance. It is now absorbed into an implicit $\mathbf{r}$ dependence in $k$.

We consider the part of Eq. (B1) proportional to the infinitesimal $\mathbf{E}(t)$. Because of the isotropy of the Fermi surface and the scattering, we then use the ansatz $\delta n(t, k, \omega)=k \cdot \varphi(t, \omega)$. We use a first-order gradient expansion in spatial and time derivatives with respect to the central coordinate, which is justified by the spatial uniformity of $\mathbf{E}(t)$ and $B$, and the slow temporal variation of $\mathbf{E}(t)$. The change in the momentum-integrated Keldysh conduction electron Green's function caused by $\mathbf{E}(t)$ through $\delta n$ then is [46]

$$
\begin{aligned}
\delta G_{K}^{c}(t, \omega) \equiv & \int d^{2} k \delta G_{K}^{c}(t, k, \omega) \\
= & -2 \int d^{2} k\left[G_{R}^{c}(|k|, \omega)-G_{A}^{c}(|k|, \omega)\right] \delta n(t, k, \omega) \\
& -2 i \int d^{2} k \partial_{\omega} \operatorname{Re}\left[G_{R}^{c}(|k|, \omega)\right] \partial_{t} \delta n(t, k, \omega) \\
& +2 i \int d^{2} k \partial_{k} \operatorname{Re}\left[G_{R}^{c}(|k|, \omega)\right] \cdot \nabla \mathbf{A}_{B}(\mathbf{r}) \\
& \cdot \partial_{k} \delta n(t, k, \omega)=0,
\end{aligned}
$$


as $G_{f}^{R, A}$ are isotropic. We have used $\nabla \delta n(t, k, \omega)=$ $\nabla \mathbf{A}_{B}(\mathbf{r}) \cdot \partial_{k} \delta n(t, k, \omega)$, due to the implicit $\mathbf{r}$ dependence in $k$. The retarded and advanced conduction electron Green's functions are not changed by the applied electric field, as they are only influenced by the change in the distribution $\delta n$ through the self-energies [46], which, as we show below, are unaffected by the applied electric field.

On the Keldysh contour, the conduction electron selfenergy is given by, analogous to Eq. (2.4),

$$
\begin{aligned}
\Sigma^{c}\left(t_{1}, t_{2}\right) & =-g^{2} G^{c}\left(t_{1}, t_{2}\right) G\left(t_{1}, t_{2}\right) G\left(t_{2}, t_{1}\right), \text { or } \\
\Sigma_{>,<}^{c}\left(t_{1}, t_{2}\right) & =-g^{2} G_{>,<}^{c}\left(t_{1}, t_{2}\right) G_{>,<}\left(t_{1}, t_{2}\right) G_{<,>}\left(t_{2}, t_{1}\right) .
\end{aligned}
$$

Using the standard relations between the $>,<$ representation and the $R, A, K$ representation $[46,66]$, the changes in the conduction electron self-energies due to $\delta n$ are then given by

$$
\begin{aligned}
\delta \Sigma_{R}^{c}\left(t_{1}, t_{2}\right)= & -\frac{g^{2}}{4} \theta\left(t_{1}-t_{2}\right) \delta G_{K}^{c}\left(t_{1}, t_{2}\right)\left[G_{K}\left(t_{1}, t_{2}\right) G_{A}\left(t_{2}, t_{1}\right)\right. \\
& \left.+G_{K}\left(t_{2}, t_{1}\right) G_{R}\left(t_{1}, t_{2}\right)\right], \\
\delta \Sigma_{A}^{c}\left(t_{1}, t_{2}\right)= & -\frac{g^{2}}{4} \theta\left(t_{2}-t_{1}\right) \delta G_{K}^{c}\left(t_{1}, t_{2}\right)\left[G_{K}\left(t_{1}, t_{2}\right) G_{R}\left(t_{2}, t_{1}\right)\right. \\
& \left.+G_{K}\left(t_{2}, t_{1}\right) G_{A}\left(t_{1}, t_{2}\right)\right], \\
\delta \Sigma_{K}^{c}\left(t_{1}, t_{2}\right)= & -\frac{g^{2}}{4} \delta G_{K}^{c}\left(t_{1}, t_{2}\right)\left[\left(G_{K}\left(t_{1}, t_{2}\right) G_{K}\left(t_{2}, t_{1}\right)\right.\right. \\
& \left.+G_{R}\left(t_{1}, t_{2}\right) G_{A}\left(t_{2}, t_{1}\right)\right],\left(t_{1}>t_{2}\right), \\
\delta \Sigma_{K}^{c}\left(t_{1}, t_{2}\right)= & -\frac{g^{2}}{4} \delta G_{K}^{c}\left(t_{1}, t_{2}\right)\left[G_{K}\left(t_{1}, t_{2}\right) G_{K}\left(t_{2}, t_{1}\right)\right. \\
& \left.+G_{A}\left(t_{1}, t_{2}\right) G_{R}\left(t_{2}, t_{1}\right)\right],\left(t_{1}<t_{2}\right),
\end{aligned}
$$

which vanish due to Eq. (B3). Here, $G_{R, A, K}$ denote the island electron Green's functions at equilibrium. Similarly, for the SYK islands, we also get $\delta \Sigma_{R, A, K}=0$, for the same reason.

The $\mathcal{O}(\mathbf{E})$ part of the rhs of Eq. (B1) then is $2\left(\Sigma_{R}^{c} \circ \delta n-\delta n \circ \Sigma_{A}^{c}\right)$. Using the $p, k, \mathbf{r}$-independence of the by-definition $t$-independent equilibrium self-energies $\Sigma_{R, A, K}^{c}$, and a first-order gradient expansion in central time derivatives, the rhs of Eq. (B1) reduces to [46]

$4 i \operatorname{Im}\left[\Sigma_{R}^{c}(\omega)\right] \delta n(t, k, \omega)+2 i \partial_{\omega} \operatorname{Re}\left[\Sigma_{R}^{c}(\omega)\right] \partial_{t} \delta n(t, k, \omega)$.

We now turn to the part of the lhs of Eq. (B1) proportional to $\mathbf{E}(t)$. Following Sec. 5.7 of Ref. [46], and noting that the Wigner transform of $\nabla+A_{B}(\mathbf{r})$ is $k$, it reduces in the first-order gradient expansion in central spatial and time derivatives to

$$
\begin{aligned}
& 2 i \partial_{t} \delta n(t, k, \omega)+2 i\left(-v_{F} \partial_{t}\left|k+\mathbf{A}_{E}(t)\right| n_{f}^{\prime}(\omega)\right. \\
& \quad+v_{F} \nabla|k| \cdot \partial_{k} \delta n(t, k, \omega) \\
& \left.\quad-v_{F} \partial_{k}|k| \cdot \nabla \mathbf{A}_{B}(\mathbf{r}) \cdot \partial_{k} \delta n(t, k, \omega)\right)
\end{aligned}
$$

After some algebra, this further reduces to

$$
\begin{gathered}
2 i \partial_{t} \delta n(t, k, \omega)+2 i v_{F} \mathbf{E}(t) \cdot \hat{k} n_{f}^{\prime}(\omega) \\
+2 i v_{F} \mathcal{B}(\hat{k} \times \hat{z}) \cdot \partial_{k} \delta n(t, k, \omega) .
\end{gathered}
$$

Then, combining this with Eq. (B6), we recover Eq. (4.6). The solution to Eq. (4.6) then shows our ansatz $\delta n(t, k, \omega)=k \cdot \varphi(t, \omega)$ to be self-consistent.

[1] B. Keimer, S. A. Kivelson, M. R. Norman, S. Uchida, and J. Zaanen, From Quantum Matter to High-Temperature Superconductivity in Copper Oxides, Nature (London) 518, 179 (2015).

[2] S. Kasahara, T. Shibauchi, K. Hashimoto, K. Ikada, S. Tonegawa, R. Okazaki, H. Shishido, H. Ikeda, H. Takeya, K. Hirata, T. Terashima, and Y. Matsuda, Evolution from Non-Fermi- to Fermi-Liquid Transport via Isovalent Doping in $\mathrm{BaFe}_{2}\left(\mathrm{As}_{1-x} \mathrm{P}_{x}\right)_{2}$ Superconductors, Phys. Rev. B 81, 184519 (2010).

[3] S. Sachdev and B. Keimer, Quantum Criticality, Phys. Today 64, No. 2, 29 (2011).

[4] V. J. Emery and S. A. Kivelson, Superconductivity in Bad Metals, Phys. Rev. Lett. 74, 3253 (1995).

[5] I. M. Hayes, R. D. McDonald, N. P. Breznay, T. Helm, P. J. W. Moll, M. Wartenbe, A. Shekhter, and J. G. Analytis, Scaling between Magnetic Field and Temperature in the High-Temperature Superconductor $\mathrm{BaFe}_{2}\left(\mathrm{As}_{1-x} \mathrm{P}_{x}\right)_{2}$, Nat. Phys. 12, 916 (2016).

[6] P. Giraldo-Gallo, J. A. Galvis, Z. Stegen, K. A. Modic, F. F. Balakirev, J. B. Betts, X. Lian, C. Moir, S. C. Riggs, J. Wu, A. T. Bollinger, X. He, I. Bozovic, B. J. Ramshaw, R. D. McDonald, G. S. Boebinger, and A. Shekhter, ScaleInvariant Magnetoresistance in a Cuprate Superconductor, arXiv:1705.05806.

[7] S. Sachdev and J. Ye, Gapless Spin-Fluid Ground State in a Random Quantum Heisenberg Magnet, Phys. Rev. Lett. 70, 3339 (1993).

[8] A. Y. Kitaev, KITP, University of California, Santa Barbara, Entanglement in Strongly-Correlated Quantum Matter (2015), http://online.kitp.ucsb.edu/online/entangled15/kitaev/.

[9] O. Parcollet and A. Georges, Non-Fermi-Liquid Regime of a Doped Mott Insulator, Phys. Rev. B 59, 5341 (1999).

[10] X.-Y. Song, C.-M. Jian, and L. Balents, Strongly Correlated Metal Built from Sachdev-Ye-Kitaev Models, Phys. Rev. Lett. 119, 216601 (2017).

[11] J. Maldacena and D. Stanford, Remarks on the Sachdev-YeKitaev Model, Phys. Rev. D 94, 106002 (2016).

[12] J. Maldacena, S. H. Shenker, and D. Stanford, A Bound on Chaos, J. High Energy Phys. 08 (2016) 106.

[13] S. Sachdev, Quantum Phase Transitions 1st ed. (Cambridge University Press, Cambridge, UK, 1999). 
[14] J. Sonner and M. Vielma, Eigenstate Thermalization in the Sachdev-Ye-Kitaev Model, J. High Energy Phys. 11 (2017) 149.

[15] J. M. Deutsch, Quantum Statistical Mechanics in a Closed System, Phys. Rev. A 43, 2046 (1991).

[16] M. Srednicki, Chaos and Quantum Thermalization, Phys. Rev. E 50, 888 (1994).

[17] S. Sachdev, Holographic Metals and the Fractionalized Fermi Liquid, Phys. Rev. Lett. 105, 151602 (2010).

[18] S. Sachdev, Strange Metals and the AdS/CFT Correspondence, J. Stat. Mech. (2010) P11022.

[19] J. Maldacena, D. Stanford, and Z. Yang, Conformal Symmetry and Its Breaking in Two Dimensional Nearly Anti-deSitter Space, Prog. Theor. Exp. Phys. 2016, 12C104 (2016).

[20] A. Kitaev and S. J. Suh, The Soft Mode in the Sachdev-YeKitaev Model and Its Gravity Dual, arXiv:1711.08467.

[21] J. Maldacena, D. Stanford, and Z. Yang, Diving into Traversable Wormholes, Fortschr. Phys. 65, 1700034 (2017).

[22] T. Faulkner, H. Liu, J. McGreevy, and D. Vegh, Emergent Quantum Criticality, Fermi Surfaces, and $\mathrm{AdS}_{2}$, Phys. Rev. D 83, 125002 (2011).

[23] S. Sachdev, Bekenstein-Hawking Entropy and Strange Metals, Phys. Rev. X 5, 041025 (2015).

[24] C. M. Varma, P. B. Littlewood, S. Schmitt-Rink, E. Abrahams, and A. E. Ruckenstein, Phenomenology of the Normal State of $\mathrm{Cu}$-O High-Temperature Superconductors, Phys. Rev. Lett. 63, 1996 (1989).

[25] M. Mitrano, A. A. Husain, S. Vig, A. Kogar, M. S. Rak, S. I. Rubeck, J. Schmalian, B. Uchoa, J. Schneeloch, R. Zhong, G. D. Gu, and P. Abbamonte, Singular Density Fluctuations in the Strange Metal Phase of a Copper-Oxide Superconductor, arXiv:1708.01929.

[26] T. Faulkner, N. Iqbal, H. Liu, J. McGreevy, and D. Vegh, Charge Transport by Holographic Fermi Surfaces, Phys. Rev. D 88, 045016 (2013).

[27] Y. Gu, X.-L. Qi, and D. Stanford, Local Criticality, Diffusion and Chaos in Generalized Sachdev-Ye-Kitaev Models, J. High Energy Phys. 11 (2017) 014.

[28] R. A. Davison, W. Fu, A. Georges, Y. Gu, K. Jensen, and S. Sachdev, Thermoelectric Transport in Disordered Metals Without Quasiparticles: The Sachdev-Ye-Kitaev Models and Holography, Phys. Rev. B 95, 155131 (2017).

[29] M. Milovanović, S. Sachdev, and R. N. Bhatt, EffectiveField Theory of Local-Moment Formation in Disordered Metals, Phys. Rev. Lett. 63, 82 (1989).

[30] R. N. Bhatt and D. S. Fisher, Absence of Spin Diffusion in Most Random Lattices, Phys. Rev. Lett. 68, 3072 (1992).

[31] A. C. Potter, M. Barkeshli, J. McGreevy, and T. Senthil, Quantum Spin Liquids and the Metal-Insulator Transition in Doped Semiconductors, Phys. Rev. Lett. 109, 077205 (2012).

[32] S. Burdin, D. R. Grempel, and A. Georges, Heavy-Fermion and Spin-Liquid Behavior in a Kondo Lattice with Magnetic Frustration, Phys. Rev. B 66, 045111 (2002).

[33] D. Ben-Zion and J. McGreevy, Strange Metal from Local Quantum Chaos, Phys. Rev. B 97, 155117 (2018).

[34] A. M. Dykhne, Anomalous Plasma Resistance in a Strong Magnetic Field, J. Exp. Theor. Phys. 32, 348 (1971).
[35] D. Stroud, Generalized Effective-Medium Approach to the Conductivity of an Inhomogeneous Material, Phys. Rev. B 12, 3368 (1975).

[36] M. M. Parish and P. B. Littlewood, Non-saturating Magnetoresistance in Heavily Disordered Semiconductors, Nature (London) 426, 162 (2003).

[37] M. M. Parish and P. B. Littlewood, Classical Magnetotransport of Inhomogeneous Conductors, Phys. Rev. B 72, 094417 (2005).

[38] V. Guttal and D. Stroud, Model for a Macroscopically Disordered Conductor with an Exactly Linear HighField Magnetoresistance, Phys. Rev. B 71, 201304 (2005).

[39] J. C. W. Song, G. Refael, and P. A. Lee, Linear Magnetoresistance in Metals: Guiding Center Diffusion in a Smooth Random Potential, Phys. Rev. B 92, 180204 (2015).

[40] N. Ramakrishnan, Y. T. Lai, S. Lara, M. M. Parish, and S. Adam, Equivalence of Effective Medium and Random Resistor Network Models for Disorder-Induced Unsaturating Linear Magnetoresistance, Phys. Rev. B 96, 224203 (2017).

[41] M. Crisan and C. P. Moca, Specific Heat of a Marginal Fermi Liquid, J. Supercond. 9, 49 (1996).

[42] A. Georges, O. Parcollet, and S. Sachdev, Quantum Fluctuations of a Nearly Critical Heisenberg Spin Glass, Phys. Rev. B 63, 134406 (2001).

[43] A. A. Patel, Download imaginary-time code, 2017, http:// qpt.physics.harvard.edu/ggc.m.

[44] A. A. Patel, Download real-time code, 2017, http://qpt .physics.harvard.edu/ggcrealtime.m.

[45] A. Éfros and M. Pollak, Electron-Electron Interactions in Disordered Systems, Modern Problems in Condensed Matter Sciences Vol. 10 (North-Holland, Amsterdam, 1985).

[46] A. Kamenev, Field Theory of Non-equilibrium Systems (Cambridge University Press, Cambridge, England, 2011).

[47] C. P. Nave and P. A. Lee, Transport Properties of a Spinon Fermi Surface Coupled to a U(1) Gauge Field, Phys. Rev. B 76, 235124 (2007).

[48] B. L. Altshuler, D. Khmel'nitzkii, A. I. Larkin, and P. A. Lee, Magnetoresistance and Hall Effect in a Disordered Two-Dimensional Electron Gas, Phys. Rev. B 22, 5142 (1980).

[49] F. Pelzer, Amplitude of the de Haas-van Alphen Oscillations for a Marginal Fermi Liquid, Phys. Rev. B 44, 293 (1991).

[50] S. A. Hartnoll, A. Lucas, and S. Sachdev, Holographic Quantum Matter, arXiv:1612.07324.

[51] A. A. Patel, R. A. Davison, and A. Levchenko, Hydrodynamic Flows of Non-Fermi Liquids: Magnetotransport and Bilayer Drag, Phys. Rev. B 96, 205417 (2017).

[52] A. Lucas, J. Crossno, K. C. Fong, P. Kim, and S. Sachdev, Transport in Inhomogeneous Quantum Critical Fluids and in the Dirac Fluid in Graphene, Phys. Rev. B 93, 075426 (2016).

[53] Holographic realizations of a variety of magnetoresistance scalings, including quadrature, were found in Ref. [54].

[54] E. Kiritsis and L. Li, Quantum Criticality and DBI Magnetoresistance, J. Phys. A 50, 115402 (2017).

[55] K. McElroy, J. Lee, J. Slezak, D.-H. Lee, H. Eisaki, S. Uchida, and J. Davis, Atomic-Scale Sources and Mechanism 
of Nanoscale Electronic Disorder in $\mathrm{Bi}_{2} \mathrm{Sr}_{2} \mathrm{CaCu}_{2} \mathrm{O}_{8+\delta}$, Science 309, 1048 (2005).

[56] T. Hanaguri, Y. Kohsaka, J. C. Davis, C. Lupien, I. Yamada, M. Azuma, M. Takano, K. Ohishi, M. Ono, and H. Takagi, Quasiparticle Interference and Superconducting Gap in $\mathrm{Ca}_{2-x} \mathrm{Na}_{x} \mathrm{CuO}_{2} \mathrm{Cl}_{2}$, Nat. Phys. 3, 865 (2007).

[57] Y. Kohsaka, T. Hanaguri, M. Azuma, M. Takano, J. C. Davis, and H. Takagi, Visualization of the Emergence of the Pseudogap State and the Evolution to Superconductivity in a Lightly Hole-Doped Mott Insulator, Nat. Phys. 8, 534 (2012).

[58] P. Zhang, Dispersive Sachdev-Ye-Kitaev Model: Band Structure and Quantum Chaos, Phys. Rev. B 96, 205138 (2017).

[59] A. Haldar, S. Banerjee, and V. B. Shenoy, Higher-Dimensional SYK Non-Fermi Liquids at Lifshitz Transitions, arXiv: 1710.00842 .
[60] J. Ping, I. Yudhistira, N. Ramakrishnan, S. Cho, S. Adam, and M. S. Fuhrer, Disorder-Induced Magnetoresistance in a Two-Dimensional Electron System, Phys. Rev. Lett. 113, 047206 (2014).

[61] N. Ramakrishnan, M. Milletari, and S. Adam, Transport and Magnetotransport in Three-Dimensional Weyl Semimetals, Phys. Rev. B 92, 245120 (2015).

[62] J. G. Analytis and B. Ramshaw (private communications).

[63] D. Chowdhury, Y. Werman, E. Berg, and T. Senthil, Translationally Invariant Non-Fermi Liquid Metals with Critical Fermi-Surfaces: Solvable Models, arXiv:1801.06178.

[64] R. B. Thomas, Magnetic Corrections to the Boltzmann Transport Equation, Phys. Rev. 152, 138 (1966).

[65] D. C. Langreth, Hall Coefficient of Hubbard's Model, Phys. Rev. 148, 707 (1966).

[66] A. Eberlein, V. Kasper, S. Sachdev, and J. Steinberg, Quantum Quench of the Sachdev-Ye-Kitaev Model, Phys. Rev. B 96, 205123 (2017). 